\title{
Revisiting the 1954 suspension experiments of R. A. Bagnold
}

\author{
By M. L. HUNT ${ }^{1}$, R. ZENIT ${ }^{2}$, C. S. CAMPBELL ${ }^{3}$ \\ AND C. E. BREN NEN ${ }^{1}$ \\ ${ }^{1}$ Division of Engineering and Applied Sciences, California Institute of Technology, Pasadena, \\ CA 91125, USA \\ ${ }^{2}$ Instituto de Investigaciones en Materiales, Universidad Nacional Autónoma de México, \\ México D.F. 04510, México \\ ${ }^{3}$ Department of Mechanical Engineering, University of Southern California, Los Angeles, \\ CA 90089-1453, USA
}

(Received 11 April 2001 and in revised form 1 August 2001)

In 1954 R. A. Bagnold published his seminal findings on the rheological properties of a liquid-solid suspension. Although this work has been cited extensively over the last fifty years, there has not been a critical review of the experiments. The purpose of this study is to examine the work and to suggest an alternative reason for the experimental findings. The concentric cylinder rheometer was designed to measure simultaneously the shear and normal forces for a wide range of solid concentrations, fluid viscosities and shear rates. As presented by Bagnold, the analysis and experiments demonstrated that the shear and normal forces depended linearly on the shear rate in the "macroviscous' regime; as the grain-to-grain interactions increased in the 'grain-inertia' regime, the stresses depended on the square of the shear rate and were independent of the fluid viscosity. These results, however, appear to be dictated by the design of the experimental facility. In Bagnold's experiments, the height $(h)$ of the rheometer was relatively short compared to the spacing $(t)$ between the rotating outer and stationary inner cylinder $(h / t=4.6)$. Since the top and bottom end plates rotated with the outer cylinder, the flow contained two axisymmetric counter-rotating cells in which flow moved outward along the end plates and inward through the central region of the annulus. At higher Reynolds numbers, these cells contributed significantly to the measured torque, as demonstrated by comparing Bagnold's pure-fluid measurements with studies on laminar-to-turbulent transitions that pre-date the 1954 study. By accounting for the torque along the end walls, Bagnold's shear stress measurements can be estimated by modelling the liquid-solid mixture as a Newtonian fluid with a corrected viscosity that depends on the solids concentration. An analysis of the normal stress measurements was problematic because the gross measurements were not reported and could not be obtained.

\section{Introduction}

The career of Ralph Alger Bagnold began in the British Royal Army, where he rose to the rank of Brigadier. Early in his career he was stationed in North Africa and the Far East, and during this time he became fascinated with the deserts of Egypt, Sudan and Libya (Bagnold 1990, Kenn 1990). His travels in the desert were 
supported in part by the Royal Geographical Society and involved explorations and mappings of the remote parts of the Libyan Desert (Bagnold 1936a). On returning to England in the mid-1930s, he began to explore the physics of sand movement and the evolution of the desert sand dunes. Although Bagnold studied engineering at Cambridge University in the early 1920s, he considered himself an amateur scientist. With some support from the hydraulics laboratory at Imperial College, he built a special wind tunnel and began to make measurements of velocity profiles and the relation between the air flow rates and the entrainment of sand. His experiments on sand both in the laboratory and in the desert led to several publications (Bagnold 1936b, 1937, 1938) and later a book entitled, The Physics of Blown Sand and Desert Dunes (Bagnold 1941), which was been reprinted several times and is due to be republished in paperback in 2002.

During World War II, Bagnold formed the Long Range Desert Group, which penetrated the Libyan Desert and provided details on enemy troop movements (Shaw 1945). Bagnold was credited with much of the Group's success because of his leadership, knowledge of the desert, and his inventions like the sun-compass, rope ladders, and steel channels for freeing vehicles from the sand (Shaw 1945; Kenn 1990). On returning to England in 1944, he found that he had been elected a Fellow of the Royal Society of London. He continued his research by examining the effects of sea waves on the erosion and formation of beaches in preparation for the allied landing on the European continent (Bagnold 1946, 1990). These initial inquiries became the foundation of Bagnold's influential career in hydraulics and sediment transport.

In his 1990 memoir entitled Sand, Wind and War: Memoirs of a Desert Explorer, Bagnold wrote that after the war he began a series of " "try-it-and-see' experiments examining how the presence of a dispersion of solid grains affects the flow of a liquid". He was curious about the "mechanism by which a stream of water transports solids along its bed at a definite rate", and envisioned that the physics was similar to that for windblown sand (see the 1954 preface to The Physics of Blown Sand and Desert Dunes). Bagnold begins his 1954 paper by referencing the work by Reynolds (1885) on the dilatancy of a granular bed and the effect of shear, and the work by Einstein (1906) on the effective viscosity of a suspension of particles. These studies were limited to low shear rates or low concentrations. Bagnold envisioned a series of carefully controlled experiments in which a known mass of particles could be dispersed uniformly in a liquid. To this end, he began to make his own particles that would be of the same density as water (Bagnold 1990). He used a 'shot tower' method that allowed a stream of molten plastic drops (in the 1954 paper, he described the material as a " $50 \%$ mixture of paraffin wax and lead stearate") to solidify as they fell through the air. He then did some preliminary experiments by varying the concentration of particles and observed that "liquid's resistance to shear increased, till finally the whole liquid-solid substance suddenly 'froze' solid".

Besides the effect of the particles on the shear stress, Bagnold intuitively understood that shearing of closely spaced particles would also generate a normal or dispersive stress. "As a snooker player, I knew that when the cue ball strikes another ball at a glancing blow, both balls move away from one another with momentum components at right angles to the original direction of the cue ball. So I expected that the random collisions that must occur when a dispersion of solids is sheared would amount to a dispersive pressure" (Bagnold 1990). Hence, Bagnold cleverly designed his experiment to measure simultaneously the shear and normal forces generated by a sheared dispersion of neutrally buoyant particles. The concentric-cylinder rheometer had an inner cylinder with a flexible membrane that allowed measurements of the 
static pressure; the outer cylinder rotated at a constant speed and was driven by a lathe (Bagnold 1954, 1990).

From the experimental measurements and from physical arguments, Bagnold identified two flow regimes. For small shear rates in viscous liquids the shear stresses behaved like a Newtonian fluid with a corrected viscosity. In this 'macro-viscous' regime, both the normal and the tangential stresses were linearly proportional to the liquid's dynamic viscosity $(\mu)$, the shear rate $(\gamma)$ and a function of the solid fraction, $\phi$. The stresses varied with the solids concentration as $\lambda^{3 / 2}$, where $\lambda$ is the 'linear concentration', defined as the ratio of the grain diameter to the mean radial separation distance. The linear concentration was related to the solid fraction by

$$
\lambda=1 /\left[\left(\phi_{o} / \phi\right)^{1 / 3}-1\right],
$$

where $\phi_{o}$ is the maximum possible concentration. Thus, both the normal and tangential stresses scaled as $\lambda^{3 / 2} \mu \gamma$. For larger shear rates or lower liquid viscosities, the material fell into the 'grain-inertia regime' in which the stresses were independent of the fluid viscosity and depended on the square of the shear rate and the square of the function $\lambda$; hence, the stresses were proportional to $\rho d^{2} \lambda^{2} \gamma^{2}$ where $\rho$ is the density (either the density of the fluid or of the solid for a neutrally buoyant suspension) and $d$ is the particle diameter. Bagnold justified this conclusion with a simple model that treated particle interactions as impacts for which the collision impulse scaled as $\rho d \gamma$ and the collision rate scaled as $d \gamma$.

Taking the ratio of these two scalings, Bagnold derived a parameter, $N$, to distinguish the flow regimes,

$$
N=\frac{\rho d^{2} \lambda^{2} \gamma^{2}}{\mu \gamma \lambda^{3 / 2}}=\frac{\rho d^{2} \lambda^{1 / 2} \gamma}{\mu} .
$$

Bagnold identified the linear macro-viscous regime with $N<40$ and the quadratic variation in the stresses with $N>450$; a transitional regime fell between these two limiting values. The parameter $N$ has since been called the Bagnold number (Hill 1966) and has been extended to dense particle flows using the particle density in place of the fluid-solid density.

Besides the distinction between the different regimes, Bagnold also examined the ratio of the shear to normal stresses for a given concentration, $T_{\lambda} / P_{\lambda}=\tan \alpha$, defining $\alpha$ as the effective friction angle. He referred to this ratio as a dynamic analogue of the static friction coefficient (Bagnold 1956). In the viscous regime the ratio was approximately equal to 0.75 , and reached a constant value of approximately 0.32 in the grain-inertia regime. Bagnold noted that the static friction angle of $32^{\circ}$ for sand corresponded to a stress ratio, $\tan \alpha=0.63$, which was intermediate to the two limiting dynamic values. In summarizing Bagnold stated, "The most important result of this rotating drum experiment was that, independent of both solids' concentration and shear rate, the resistance attributable to the solids was always on the order of half of the dispersive pressure. That is, the coefficient of solid to solid friction for a sheared dispersion of granular solids is virtually the same as that for the same solids in continuous contact" (Bagnold 1990).

In the 1954 paper, the results of the measurements were applied to three problems the flow of dry sand under gravity, size sorting of grains, and flows of river gravel. In Bagnold's later work on bed load transport in streams and rivers, the values for the stress ratio were used extensively to determine the critical conditions under which a particle remained suspended (Bagnold 1956, 1966a, 1973, 1980). In addition, Bagnold used the results to explain the source of booming sounds associated with 


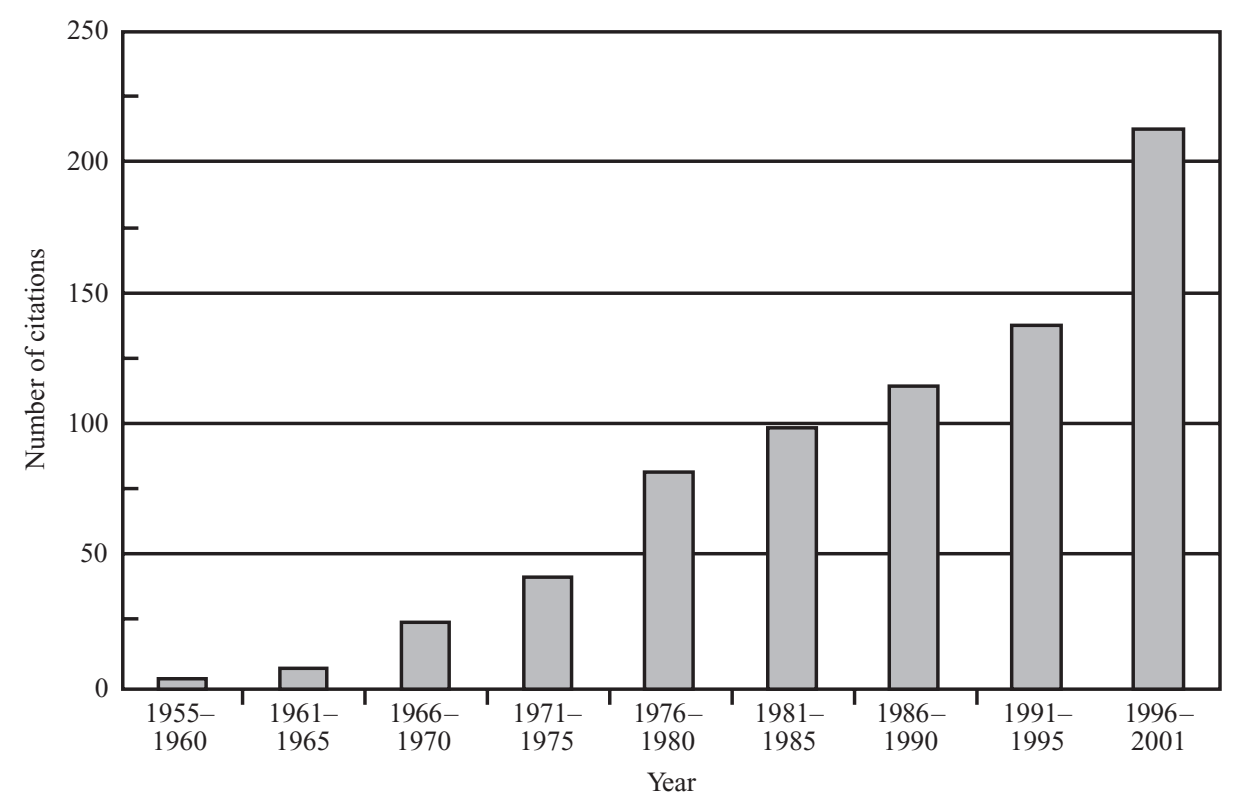

FIGURE 1. Number of citations to Bagnold's (1954) paper since publication.

the movement of sand dunes in the desert (Bagnold 1966b), and to Æolian transport on Mars (Sagan \& Bagnold 1975). A bibliography of Bagnold's work can be found in the short biography by Thorne \& Soar (1996) or in the biographical memoir by Kenn (1990).

The results of Bagnold's 1954 rheological experiments have been referenced in hundreds of studies reported in the scientific literature as illustrated in figure 1. This figure is based on citations tabulated in the Institute for Scientific Information Citation Database. In total, the paper has been referenced approximately 725 times in archival journals over the last 46 years (up to March, 2001). The companion study that was published in 1956 and entitled "The flow of cohesionless grains in fluids" has been referenced approximately 350 times. In some of these papers, there is only a passing reference to Bagnold's work. However, several papers have tried to repeat or to simulate the experimental results, and the constitutive relations have been applied to a range of fluid-solid flows (for example Savage \& Jeffrey 1981; Savage \& McKeown 1983; Hanes \& Inman 1985; Shibata \& Mei 1986; Prasad \& Kytömaa 1995; Hutter \& Rajagopal 1994; Potapov, Hunt \& Campbell 2001). Although the research has had tremendous impact on the fields of granular materials, debris flows, slurry and sediment transport, there has not been a critical analysis of the experiments.

\section{Re-examination of the experiments}

A sketch of Bagnold's experimental apparatus is shown in figure 2. The radii of the outer and inner cylinders were $r_{o}=5.7 \mathrm{~cm}$ and $r_{i}=4.62 \mathrm{~cm}$, respectively; hence, the ratio of gap, $t=r_{o}-r_{i}$, to outer cylinder was $t / r_{o}=0.19$. The height, $h$, of the test section was $5 \mathrm{~cm}$ and the corresponding ratio of height to gap was $h / t=4$.6. The outer cylinder of the rheometer was rotated at a constant rate ranging from 15 to approximately 500 r.p.m. The inner cylinder was held fixed using a spring that enabled a measurement of the torque. The reported net shear stress on the inner wall, $T_{\lambda}$, 


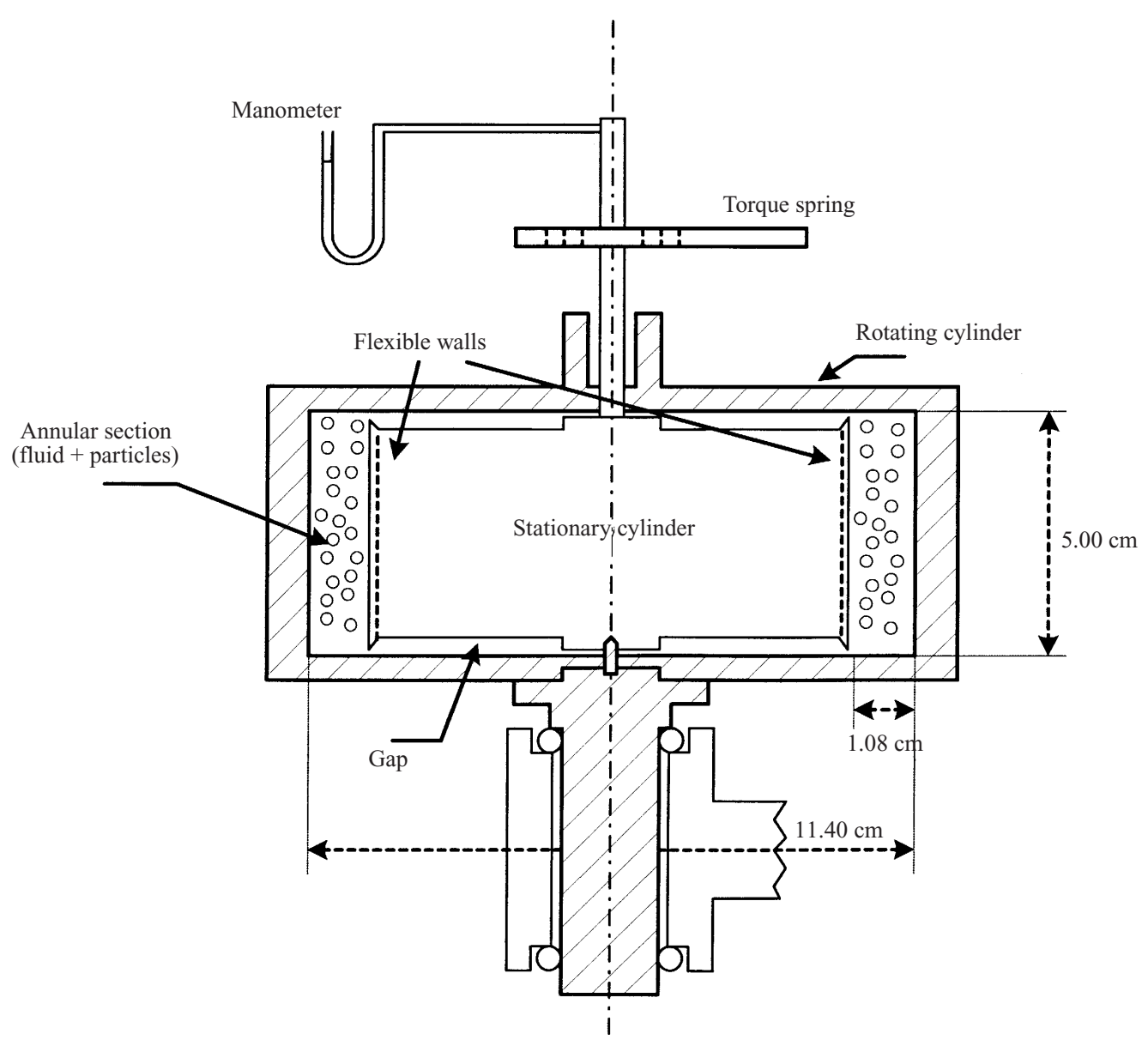

FiguRE 2. Experimental apparatus used by Bagnold (1954) to measure the shear and normal forces in a sheared suspension.

ranged from 0.3 to $60 \mathrm{~N} \mathrm{~m}^{-2}$; hence the net torques $\left(2 \pi r_{i}{ }^{2} h T_{\lambda}\right)$ ranged from $2 \times 10^{-4}$ to $4 \times 10^{-2} \mathrm{~N} \mathrm{~m}$. The sidewall of the inner cylinder was made from sheet rubber that would deflect in response to the applied pressure. The volume behind the membrane was filled with a fluid that was free to rise in a vertical tube, and the net normal stress, $P_{\lambda}$, was determined using an inclined manometer. The normal stress varied from 2.5 to $200 \mathrm{~N} \mathrm{~m}^{-2}$, which translated into a water column height from 0.025 to $2.0 \mathrm{~cm}$. The accuracies of the measurements were not reported. Note that the notation $T$ and $P$ follows from the paper by Bagnold and represents net stresses determined from averaged force measurements as described in detail in this section.

The experiment used $1.3 \mathrm{~mm}$ diameter wax spheres suspended in water or in a similar density mixture of glycerin, water and alcohol. The experiments were conducted at 10 different concentrations of solids ( $\lambda$ from 1.3 to 17 corresponding to solid fractions from $\phi=0.135$ to 0.623 with $\phi_{o}=0.74$ as used by Bagnold) in water and for one concentration $(\lambda=11, \phi=0.555)$ in the glycerin mixture. A listing of the experimental data sets is given in table 1, including the experimental ranges of Bagnold and Reynold numbers based on gap spacing $\left(R e=\rho r_{o} \omega t / \mu\right.$ with $\mu$ the dynamic viscosity of the liquid). For the lowest concentrations, the pressures were not 


\begin{tabular}{|c|c|c|c|c|c|c|c|c|}
\hline$\lambda$ & $\phi$ & Fluid & $\begin{array}{l}\text { Normal }(\mathrm{P}) \\
\text { or Shear }(\mathrm{T})\end{array}$ & $\begin{array}{l}\text { Bagnold } \\
\text { number, } N\end{array}$ & $\begin{array}{c}\text { Reynolds } \\
\text { number, Re }\end{array}$ & $C_{1}$ or $C_{2}$ & $m$ or $n$ & $r$ \\
\hline 1.3 & 0.135 & $\mathrm{~W}$ & $\mathrm{~T}$ & $237-308$ & $14000-18000$ & $1.3 \times 10^{-2}$ & 1.6 & 1.0 \\
\hline 2.1 & 0.222 & W & $\mathrm{T}$ & $110-620$ & $5100-29000$ & $8.0 \times 10^{-4}$ & $1.6 \pm 0.4$ & 1.0 \\
\hline \multirow[t]{2}{*}{3.1} & 0.308 & $\mathrm{~W}$ & $\mathrm{P}$ & 615 & 23000 & & & \\
\hline & & & $\mathrm{T}$ & $130-750$ & $5700-29000$ & $2.9 \times 10^{-3}$ & $1.5 \pm 0.2$ & 0.998 \\
\hline \multirow[t]{2}{*}{4.1} & 0.375 & $\mathrm{~W}$ & $\mathrm{P}$ & $220-870$ & $7100-29000$ & $4.1 \times 10^{-5}$ & $2.1 \pm 0.3$ & 0.998 \\
\hline & & & $\mathrm{T}$ & $150-870$ & $5100-29000$ & $2.8 \times 10^{-3}$ & $1.6 \pm 0.1$ & 0.999 \\
\hline \multirow[t]{2}{*}{5.7} & 0.445 & W & $\mathrm{P}$ & $180-1020$ & $5100-29000$ & $5.7 \times 10^{-4}$ & $2.0 \pm 0.3$ & 0.993 \\
\hline & & & $\mathrm{T}$ & $180-1020$ & $5100-29000$ & $3.8 \times 10^{-3}$ & $1.6 \pm 0.3$ & 0.993 \\
\hline \multirow[t]{2}{*}{7.6} & 0.495 & $\mathrm{~W}$ & $\mathrm{P}$ & $110-960$ & $2700-23000$ & $1.2 \times 10^{-3}$ & $1.9 \pm 0.1$ & 0.998 \\
\hline & & & $\mathrm{T}$ & $55-1180$ & $1300-29000$ & $2.4 \times 10^{-1}$ & $1.5 \pm 0.1$ & 0.996 \\
\hline \multirow[t]{2}{*}{9.4} & 0.532 & $\mathrm{~W}$ & $\mathrm{P}$ & $23-1070$ & $5100-24000$ & $1.9 \times 10^{-3}$ & $1.8 \pm 0.2$ & 0.998 \\
\hline & & & $\mathrm{T}$ & $230-830$ & $5100-18000$ & $7.6 \times 10^{-3}$ & $1.6 \pm 0.2$ & 0.999 \\
\hline \multirow[t]{2}{*}{11} & 0.555 & $\mathrm{~W}$ & $\mathrm{P}$ & $130-900$ & $2700-18000$ & $1.9 \times 10^{-3}$ & $1.9 \pm 0.2$ & 0.998 \\
\hline & & & $\mathrm{T}$ & $66-690$ & $1340-14000$ & $4.0 \times 10^{-2}$ & $1.5 \pm 0.1$ & 0.998 \\
\hline \multirow[t]{2}{*}{14.5} & 0.606 & W & $\mathrm{P}$ & $100-510$ & $1800-9000$ & $2.3 \times 10^{-2}$ & $1.7 \pm 0.4$ & 0.995 \\
\hline & & & $\mathrm{T}$ & $76-510$ & $1340-9000$ & $3.1 \times 10^{-2}$ & $1.6 \pm 0.2$ & 0.997 \\
\hline \multirow[t]{2}{*}{17} & 0.623 & W & $\mathrm{P}$ & $52-230$ & $840-3700$ & $1.2 \times 10^{-1}$ & $1.7 \pm 0.4$ & 0.997 \\
\hline & & & $\mathrm{T}$ & $52-310$ & $840-5100$ & $1.7 \times 10^{-1}$ & $1.6 \pm 0.2$ & 0.997 \\
\hline \multirow[t]{2}{*}{11} & 0.555 & G & $\mathrm{P}$ & $6-170$ & $120-3340$ & $4.6 \times 10^{-1}$ & $1.2 \pm 0.1$ & 0.996 \\
\hline & & & $\mathrm{T}$ & $6-50$ & $120-1000$ & $8.7 \times 10^{-1}$ & $1.1 \pm 0.1$ & 0.999 \\
\hline \multirow[t]{2}{*}{0} & 0 & W & $\mathrm{P}$ & & not reported & & & \\
\hline & & & $\mathrm{T}$ & & $8000-33000$ & $2.0 \times 10^{-5}$ & 2.0 & \\
\hline
\end{tabular}

TABLE 1. Bagnold's experimental parameters

reported. For some data sets at a fixed concentration, Bagnold used a different range of Reynolds numbers for the shear and normal stresses. In addition, he reported the experimental results for the shear stress for pure water $(\lambda=0)$. Note that the concentrations listed in table 1 are taken directly from Bagnold's paper; except for $\lambda=1.3,14.5$ and 17, the reported values of $\phi$ are somewhat lower than would be calculated using $\phi_{o}=0.74$ in equation (1.1).

In figure 3 , the non-dimensional shear $T_{\lambda}\left[\rho d^{2} /\left(\lambda \mu^{2}\right)\right]$ and normal stress $P_{\lambda}\left[\rho d^{2} /\left(\lambda \mu^{2}\right)\right]$ are plotted as a function of Bagnold number for all concentrations. In Bagnold's paper, a similar figure is shown but only for the data corresponding to $\lambda=11$. The shear and normal stresses collapse to separate curves for the lower concentrations $(\lambda<12)$. The stresses for the highest concentrations $(\lambda=14.5$ and 17) are slightly greater. From the physical arguments described in the context of equation (1.2), Bagnold proposed correlations for the two limiting cases. For the grain-inertia regime with $\lambda<12$, the normal and shear stresses were expressed as follows:

$$
P_{\lambda}=0.042 \rho(\lambda \gamma d)^{2} \cos \alpha,
$$

and

$$
T_{\lambda} / P_{\lambda}=\tan \alpha_{1}
$$

where $\tan \alpha_{1}=0.32$ (in Bagnold 1954, the density $\rho$ is missing from (2.1); however, in the 1956 paper, the density is included). The non-dimensional pressure in the grain inertia regime depended quadratically on the Bagnold number,

$$
P_{\lambda} /\left[\mu^{2} \lambda /\left(\rho d^{2}\right)\right]=0.040 N^{2} .
$$




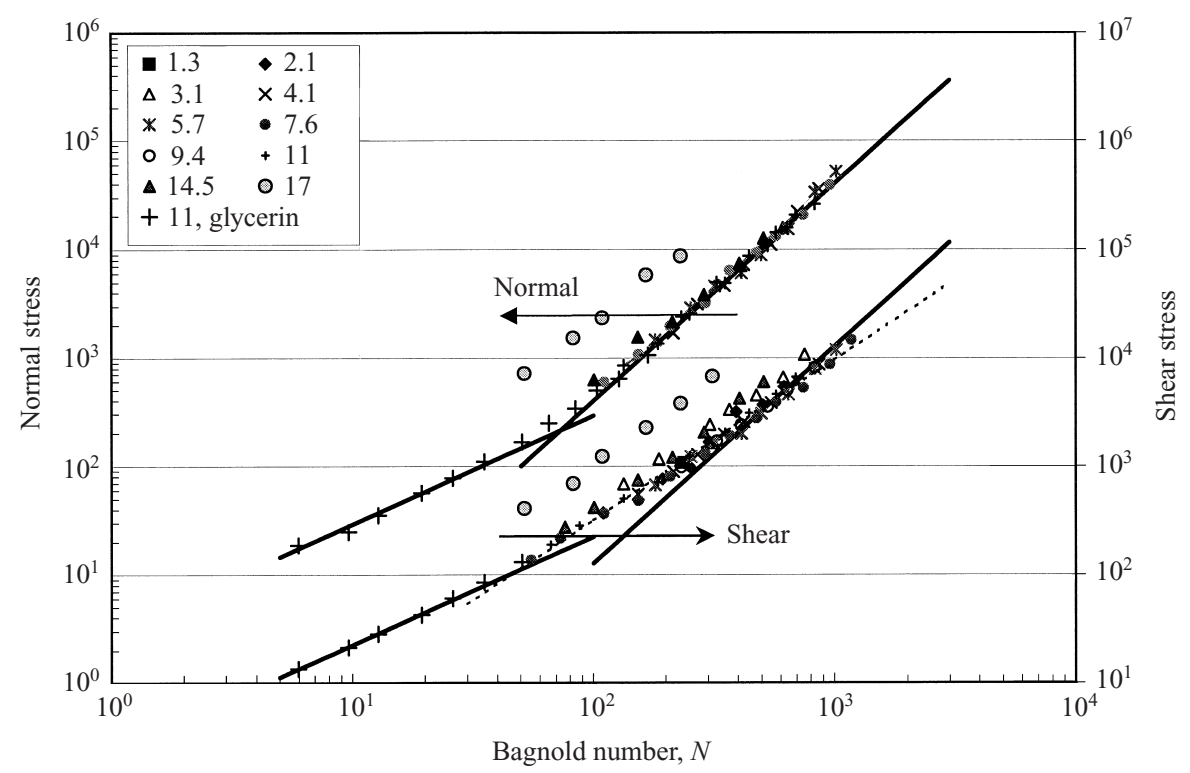

FIGURE 3. Experimental shear and normal stresses as reported by Bagnold (1954) for linear concentrations $\lambda=1.3(\phi=0.135), 2.1(\phi=0.222), 3.1(\phi=0.308), 4.1(\phi=0.375), 5.7(\phi=0.445)$, $7.6(\phi=0.495), 9.4(\phi=0.532), 11(\phi=0.555), 14.5(\phi=0.606)$ and $17(\phi=0.623)$ in water and for $\lambda=11(\phi=0.555)$ in the glycerin mixture. The solid lines are the suggested correlations by Bagnold, and the dotted line is the best fit to the shear stress measurements in water.

For the macro-viscous regime, Bagnold proposed the following relations for the shear and normal stresses:

$$
\begin{aligned}
& T_{\lambda}=2.25 \lambda^{3 / 2} \mu \gamma, \\
& P_{\lambda}=T_{\lambda} / \tan \alpha_{2},
\end{aligned}
$$

where $\tan \alpha_{2}=0.75$. In terms of the Bagnold number, the non-dimensional shear stress for the macro-viscous regime follows as

$$
T_{\lambda} /\left[\mu^{2} \lambda /\left(\rho d^{2}\right)\right]=2.25 N \text {. }
$$

The expressions for the shear and normal stress in both the grain-inertia and macroviscous regimes (2.2), (2.3), (2.5), (2.6) are shown in figure 3. Note that the curve for the shear stress in the grain-inertia regime does not adequately represent the trend in the data.

For each of the experimental data sets listed in table 1, a least-squares power law relation was fitted to the data in the form of $P_{\lambda} /\left[\mu^{2} /\left(\rho d^{2}\right)\right]=C_{1} R e^{m}$ and $T_{\lambda} /\left[\mu^{2} /\left(\rho d^{2}\right)\right]=C_{2} R e^{n}$ over the range of Reynolds number examined for each experiment. The choice of the Reynolds number to correlate the data is explained in $\S 4$. Table 1 lists the coefficients $C_{1}, C_{2}, m, n$ and the correlation coefficient $r$ for the fit; in addition, the uncertainty in the coefficients $m$ and $n$ are given based on a $95 \%$ confidence interval. Although the pressure exponents are approximately $m=2$ for all of the water data, the shear stress exponents are closer to $n=1.5$.

Using all of the shear-stress data points for water except at the highest concentration (66 data points; $55<N<1180 ; 1.3 \leqslant \lambda \leqslant 14.5$ ), the data are best fitted by

$$
T_{\lambda} /\left[\mu^{2} \lambda /\left(\rho d^{2}\right)\right]=0.35 N^{1.48} \text {. }
$$

This correlation is shown by the dotted line in figure 3 and clearly displays a weaker 
dependence on the Bagnold number than suggested by the relations given by equation (2.2) using (2.1). A correlation for the pressure data (47 data points; $76<N<1070$; $1.3 \leqslant \lambda \leqslant 14.5$; water) results in the following relation:

$$
P_{\lambda} /\left[\mu^{2} \lambda /\left(\rho d^{2}\right)\right]=0.056 N^{1.95},
$$

which corresponds well with the dependence suggested in equation (2.1). Therefore, the shear and normal forces do not display the same dependence on the shear rate over similar ranges in $N$, contrary to the predictions of Bagnold's analysis.

For the macro-viscous regime, the only data are for the glycerin-water-alcohol mixture at $\lambda=11$, which included seven shear stress measurements between $5<N<$ 50. These data correspond well with equation (2.6). The linear and the $N^{1.5}$ shear stress curves intersect at approximately $N=50$; hence, none of the glycerin data show the higher Bagnold number dependence, and none of the water data display the linear dependence. For the normal stress data, Bagnold presented 12 measurements with glycerin for $5<N<170$. The intersection of equations (2.3) and (2.5) occurs at approximately $N=100$. At the highest Bagnold numbers for glycerin, $N=103$, 127 , and 169 , the pressure measurements do indicate a stronger dependence on the Bagnold number than displayed by the lower Bagnold number data. Therefore, with the exception of three data points for the highest pressure measurements in the glyerin mixture, Bagnold's data can be divided into two groups based on the interstitial fluid; the data obtained using water are represented well by the correlations given in equations (2.7) and (2.8), and the glycerin data are represented by equations (2.5) and (2.6).

As noted earlier, Bagnold suggested that the most important result was the ratio of the shear to normal stresses. These values are shown in figure 4 and range from 0.8 to 0.25 . Because the stresses have different dependences on the Bagnold number, the stress ratio appears to decrease with increasing Bagnold number. The limiting values suggested by Bagnold of 0.32 and 0.75 are also shown, which do not represent the data well except at the lowest Bagnold numbers.

The stresses and the stress ratios presented in figures 3 and 4 are net stresses due to the action of the particles, and are determined from measurement of the gross torque obtained minus the friction and drag associated with the experimental device. In addition, Bagnold argued that in the grain-inertia regime a distinction should be made between contributions to the torque from the fluid and solid phases. Bagnold viewed the 'grain stress' as a particle effect that conformed to the scaling described by equations (2.1) and (2.2). In addition, he noted that a fluid contribution may "persist by virtue of a residual fluid turbulence". Bagnold did not provide further arguments regarding the distinction; however in his 1956 and $1966 b$ papers, he again restated the need to separate the contributions. The notion of separating the stress into fluid and solid contributions first appeared as the 'effective stress' principle in soil mechanics and was proposed by K. Terzaghi in 1925 (see Scott 1963) for static soils saturated with a fluid. Although he did not reference Terzaghi's work, Bagnold referred to interactions with Terzaghi in describing the flow of gravel (Bagnold 1954) and later referenced Terzaghi's soil mechanics text (Terzaghi 1943) in his $1966 b$ paper.

As a result, the gross torque for the grain-inertia regime was the sum of four contributions: (i) the torque caused by spindle friction, $M_{\text {frict }}$; (ii) the torque generated by the flow in the upper and lower gap formed by the top and bottom surfaces of the rotating drum, $M_{g a p}$ (see figure 2); (iii) the torque from the fluid drag on the cylindrical surface of the drum, $M^{\prime}$; and (iv) the torque from the grain drag on the cylindrical surface, $M_{\lambda}$. As a result, the reported values for the shear stress $T_{\lambda}$ at a 


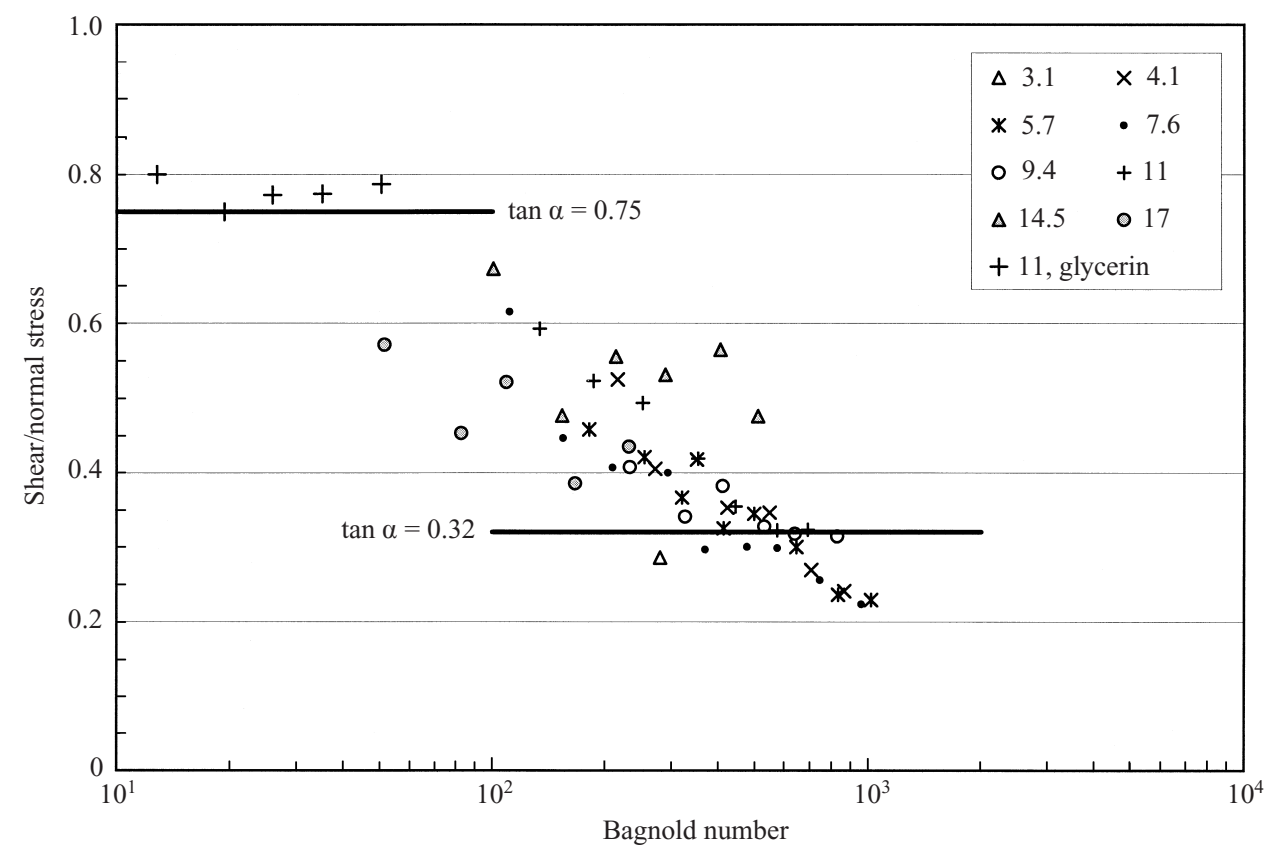

FIGURE 4. Ratio of shear stress to normal stress as reported by Bagnold (1954) for various values of $\lambda$ in water and for $\lambda=11$ in the glycerin mixture.

concentration $\lambda$ were determined from the torque $M_{\lambda}$ based on

$$
M_{\lambda}=M_{\text {gross }}-M_{\text {frict }}-M_{\text {gap }}-M^{\prime} .
$$

A series of experiments were run to determine the contributions to equation (2.9). Bagnold indicated that the gap torque, $M_{\text {gap }}$, was determined from the difference in torques when the liquid filled the end gaps and when air was locked in the gaps. Although not stated, the frictional contribution to the torque could have been estimated by operating the system dry. Bagnold asserted that the contributions to the torque from the spindle friction and from the end gaps were unchanged when particles were placed in the device. Additional measurements were also made of the torque and shear stress without particles.

To account for the fluid contribution, $M^{\prime}$, Bagnold hypothesized that it should approach the torque for a pure fluid, $M_{0}$, as the concentration was reduced to zero. In addition, $M^{\prime}$, should become progressively less important as the concentration increased because the particles should reduce the turbulence and decrease the amount of fluid within the volume. As an approximation, Bagnold assumed a simple relation for the fluid contribution to the torque, and hence the shear stress, when particles were present,

$$
M^{\prime}=M_{0} /(\lambda+1) \quad \text { and } \quad T^{\prime}=T_{0} /(\lambda+1)
$$

where the pure fluid values are $T_{0}$ and $M_{0}$. This approximation is heuristic, and Bagnold gave no clue about the origin of equation (2.10). It could be viewed as the simplest functional form to meet the desired trends. This representation was used for all of the experiments within the grain-inertia regime. For the macro-viscous flows, the distinction was not made, and the net stress written in equation (2.9) was the 


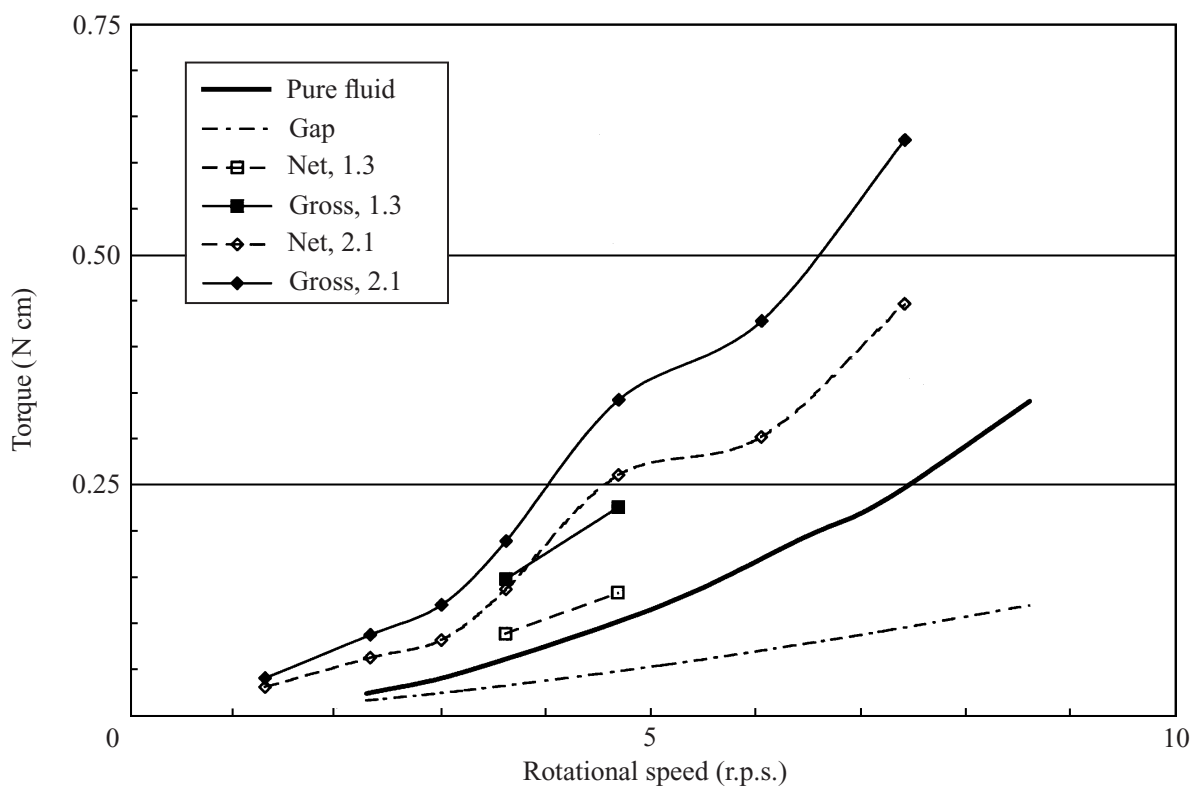

Figure 5. Contributions to the gross torque for a pure fluid and for solid concentrations of $\lambda=1.3$ and 2.1 .

difference between the gross measurement and the contributions from the friction and the flow in the gap.

Figure 5 shows the values of the pure fluid torque, $M_{0}$, calculated from the reported values of shear stress (as listed in table 1) for rotational speeds from 2.3 to 8.6 r.p.s. The measurements were not given for the lower rotational speeds because the torques were presumably below the measurable range of the rheometer. Since Bagnold did not report his raw torque data, it is illustrative to estimate the magnitude of the contributions to the torque that were subtracted by Bagnold from the gross value. Although the contribution of friction was dependent on the experimental design, the contribution from the fluid in the gaps can be estimated from a model of a rotating disk in a stationary housing (Schlichting 1955). The torque acting on a rotating disk, $M_{\text {gap }}$, can be found from $M_{\text {gap }}=(1 / 4) C_{m} \rho \omega^{2} r_{i}^{5}$, where $C_{m}$ is the moment coefficient (the factor of $1 / 4$ rather than $1 / 2$ is due to the two surfaces of the rotating disk). For laminar flow with Reynolds numbers $\left(R e_{i}=\rho r_{i}^{2} \omega / \mu\right)$ from $1 \times 10^{4}$ to $2 \times 10^{5}$, the moment coefficient can be approximated as $C_{m}=2.67 R e_{i}{ }^{-1 / 2}$, which is independent of the gap size as long as the gap is significantly smaller than the radius of the disk. The resulting torque is plotted as a function of rotational speed. Figure 5 also shows the net torque due to particle interactions for the case of $\lambda=1.3, M_{1.3}$. An additional curve is shown for the net torques calculated from the sum of the particle, gap and fluid contributions, $M_{1.3}+M_{g a p}+M^{\prime}$ with $M^{\prime}=M_{0} /(1+1.3)$. For $\lambda=1.3$, the net torque is approximately $60 \%$ of the gross torque, not including the contribution from spindle friction. A similar calculation is also shown for $\lambda=2.1$. For this concentration, the net torque is approximately $70 \%$ of the gross torque.

Besides correcting the shear stress, Bagnold also corrected the normal stress measurements. The normal stress, $P_{\lambda}$, at a given rotational speed was calculated from the difference between a gross measurement of the pressure with particles, $P_{\text {gross }}$, 
and the measurement of the pressure without particles, $P_{0}$,

$$
P_{\lambda}=P_{\text {gross }}-P_{0} .
$$

Bagnold stated that when the outer drum was rotated the pure fluid pressure increased because of "the rotation of the fluid in the end-space [see figure 2], from the axis out to the radius of the inner drum periphery". Because of this centrifugal pumping, the pure fluid pressure varied with rotational speed. However, Bagnold did not report the values, making it impossible to reconstruct his raw measurements.

\section{Bagnold's torque measurements for pure water}

Prior to 1954, several studies examined single-phase flow between a rotating outer cylinder and stationary inner cylinder (Couette 1890; Mallock 1896; Wendt 1933; Taylor 1936a, $b$; see the review by Koschmieder 1993). Although Bagnold did not compare his pure fluid results with these studies, the experimental parameters are similar and a comparison demonstrates the impact of Bagnold's experimental apparatus on his stress measurements.

The laminar tangential velocity distribution, $u_{\theta}$, for flow between infinitely long cylinders as a function of radial position, $r$, can be determined by integrating the Navier-Stokes equations (Schlichting 1955),

$$
\frac{u_{\theta}}{\omega r_{o}}=\frac{\left(r_{o} r-r_{i}^{2} r_{o} / r\right)}{\left(r_{o}^{2}-r_{i}^{2}\right)} .
$$

The torque for shear flow in a gap, $M_{\text {shear }}$, is

$$
M_{\text {shear }}=4 \pi \mu h \omega \frac{r_{o}^{2} r_{i}^{2}}{r_{o}^{2}-r_{i}^{2}} .
$$

Couette used a concentric cylinder device to measure the dynamic viscosity of water and showed that the torque depended linearly on the rotational speed of the outer cylinder (Couette 1890), as shown in equation (3.2). Above a certain critical speed, the torque increased at a faster rate. Several years later, Mallock (1896) made similar experiments, including some in which the inner cylinder rotated while the outer cylinder remained at rest. As those of Couette, these experiments showed that the flow with an outer rotating cylinder and a stationary inner cylinder was unstable beyond a certain speed. When the inner cylinder was rotated, the flow became unstable at all rotational speeds.

The Couette and Mallock research became the basis of the well-known stability studies of Taylor (1923). Taylor predicted the critical rotational speeds at which the flow became unstable for different combinations of the rotational and geometric parameters. For a rotating outer cylinder with an inner cylinder at rest, Taylor concluded that the flow was always stable. These results were in contrast with the earlier experiments by Couette (1890) and Mallock (1896).

Approximately 10 years later, separate studies by Wendt (1933) and by Taylor $(1936 a, b)$ measured the torque and the critical rotational speeds corresponding to the transition from laminar to turbulent flow for an outer rotating cylinder and an inner cylinder at rest. The Wendt and Taylor studies showed that there was a critical Reynolds number based on gap spacing $\left(R e=\rho r_{o} \omega t / \mu\right)$ above which the flow transitioned to a turbulent flow; the instability was initiated by the flow along the end plates of the annulus. The critical Reynolds number depended on the ratio of 
the gap spacing to outer radius $\left(t / r_{o}\right)$. A later study by Schultz-Grunow (1959) used linear stability theory to predict a critical Reynolds number of $R e_{c}=1670$ for $t / r_{o}$ from $10^{-3}$ to $10^{-1}$. For gap spacings greater than 0.1, the critical Reynolds number increased significantly from the limiting planar value. Later flow visualization work by Coles (1964) described the transition as 'catastrophic' with the flow consisting of a spiral band of turbulence rotating at the mean angular velocity of the two cylinders.

For the radius ratio in Bagnold's study $\left(t / r_{o}=0.19\right)$ the critical Reynolds number was approximately 18000 (Wendt 1933; Taylor 1936a; Schultz-Grunow 1959). As noted above, Bagnold presented measurements of the pure fluid shear stress for rotational speeds from 2 to 8.6 r.p.s. corresponding to $R e$ from 8800 to 33000 . Since the shear stress varied with the square of the shear rate, Bagnold concluded that the flow was turbulent over the entire range. Hence, the flow in Bagnold's work transitioned at a significantly lower Reynolds number than would have been predicted based on the earlier studies.

Besides the critical Reynolds numbers, the torque measurements can also be compared. Figure 6 shows the torque measurements for a pure fluid by Bagnold scaled by the torque computed for laminar flow between concentric cylinders as given in equation (3.2). As shown in figure 6, the Bagnold data range from 4 to 16 times larger than the torque for laminar shear flow. The figure also presents two data sets with similar geometric parameters from the work by Taylor (1936a) and by Wendt (1933). The data from Taylor are for $t / r_{o}=0.15$ and 0.21 , which bound the geometric ratio in Bagnold's study. The Taylor data are close to the laminar flow results until the critical Reynolds number (approximately 10000 for $t / r_{o}=0.15$ and 20000 for $\left.t / r_{o}=0.21\right)$ is reached. Beyond the critical Reynolds number, the data for the two experiments are comparable, and show that the torque increases with the square of the shear rate. In the Wendt study $\left(t / r_{o}=0.15\right)$, there was a free surface at the top of the annulus, and the conditions at the bottom of the annulus were varied. The Wendt data shown in figure 6 are for two configurations: (i) an end plate that rotated with the outer cylinder, and (ii) an end plate at rest. The data with the constrained end plate decreased from the laminar flow result as the Reynolds number was increased up to the critical Reynolds number (the decrease was presumably due to the movement of the free surface away from the inner cylinder by centrifugal force, reducing the contact area). With the transition to turbulent flow, an abrupt jump in the torque occurred. The critical Reynolds number for the constrained end plate corresponded well with the data by Taylor at $t / r_{o}=0.15$. When the bottom end plate co-rotated with the outer cylinder, the torque increased above the laminar flow result prior to transition, and then abruptly increased at approximately the same critical Reynolds number. Both of these data sets lie below the Bagnold data.

Although the ratios of the gap to outer radius are similar in the three studies, the height-to-gap ratios differ considerably. In the data from Taylor, the ratios were $h / t=99$ and 141. In Wendt, the ratio of the height from the end plate to the edge of the free surface to the gap was 28 . As the height of the annulus was decreased, the secondary flow associated with the ends had an increasing impact on the measured torque.

The effect of the end plates was recognized in the early work of Couette (1890) and Mallock (1896). Couette placed a guard cylinder above and below the experimental cylinder to isolate the effects of three-dimensional flow at the ends. Mallock also used a lower guard cylinder with a free surface on the upper plane. In addition, he placed mercury in the lower portion of the annulus to reduce the radial velocity of the fluid near the end plate. However, Taylor (1923) criticized the experiments 


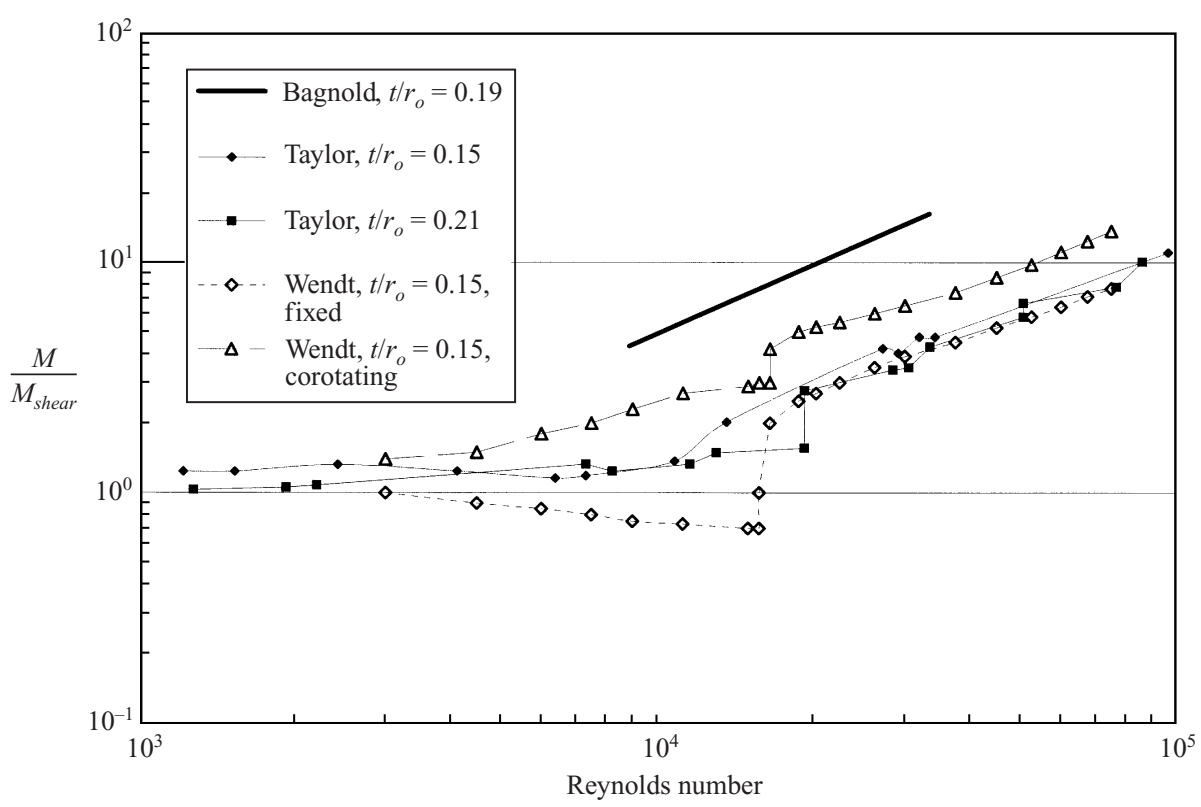

FIGURE 6. The ratio of the measured torque to torque for laminar shear flow in an annulus. Comparison between pure fluid data of Bagnold (1954) and the studies by Wendt (1933) and Taylor $(1936 a)$.

by Mallock because of the relatively short annulus $\left(h / t=8.8 ; t / r_{o}=0.23\right)$, even with the provisions of the guard cylinder and the mercury. The Taylor (1936b) paper presented the first measurements of the velocity profiles within the annulus, which showed a significant variation from the profile predicted by equation (3.1) because of the secondary circulation within the annulus. Taylor closed his paper (Taylor 1936b) by noting that he had recently read the paper by Wendt (1933), but wrote that "only qualitative results could be expected" because the height of the cylinder was not sufficiently greater than the outer diameter of the rotating cylinder. However, as shown in figure 6 , the torques were comparable.

As the height to gap ratio is decreased, it is expected that the circulation of the cells would increase with an outwardly directed flow along the ends and a inwardly directed flow through the central region. For short annuli, the two counter-rotating cells might appear to be similar to the Taylor-vortex flows found when the inner cylinder is rotated and the outer cylinder is stationary. In fact, Bagnold noted that he measured the torque (although no values are given) with the inner cylinder rotating and the outer cylinder stationary. He observed, "Though the pressures could not now be measured, the drag values, adjusted for in the same way as before, were not appreciably different, in spite of the greatly increased value of $r$ ". Although not shown in figure 6 , the torque data of Bagnold correspond closely with the measurements by Taylor (1936a) for a flow with an inner rotating cylinder and with the outer cylinder at rest (referred to as a Taylor-vortex flow; Koschmieder 1993).

\section{Examining Bagnold's data in terms of an effective viscosity}

The measurements by Bagnold for a pure fluid, and the comparisons given above, suggest that the secondary circulation had a significant effect on the torque measure- 
ments. Hence, it is of interest to examine whether the trends in the Bagnold's data with particles can be anticipated based on single-phase flow calculations.

In Bagnold's experiment, the outer cylinder was rotated along with the upper and lower boundaries of the experimental apparatus. Hence, the torque experienced by the inner cylinder resulted from the torque on the outer boundary plus the contribution to the torque from the rotating top and bottom plates. The studies by Gelhar, Schriek \& Benham (1966) for an annulus with counter-rotating cylinders and by Greenspan (1965) for a closed rotating cylinder described the flow on the end plates as an outwardly directed boundary layer. Therefore, an estimate of the torque along the end plates can be obtained from theoretical boundary layer relations for flow over a spinning disk (Schlichting 1955). In a boundary flow induced by a spinning disk, the thickness of the boundary layer is constant, $\delta \approx 4[\mu /(\rho \omega)]^{1 / 2}$; the wall shear stress $\tau_{z \theta}$ evaluated from the gradient of the tangential velocity component, $u_{\theta}$, at the wall varies linearly with radial position $(z$ is the axial distance from mid-height of the annulus). From exact solutions of the boundary layer equations, the shear stress is $\tau_{z \theta}=-\rho r\left(\mu \omega^{3} / \rho\right)^{1 / 2} C^{\prime}(0)$, where $C^{\prime}(0)$ is equal to 0.616 for laminar flow. The torque over the two end plates can then be estimated by integrating the shear stress,

$$
M_{b l} \approx-4 \pi \int_{r_{i}}^{r_{o}} r^{2} \tau_{z \theta} \mathrm{d} r=\pi \rho\left(\mu \omega^{3} / \rho\right)^{1 / 2} C^{\prime}(0)\left(r_{o}^{4}-r_{i}^{4}\right) .
$$

Hence, the torque depends on the rotational speed raised to the 1.5 power.

Recall that Bagnold's shear stress measurements also show a dependence on the rotational speed to the 1.5 power. Figure 7 is a plot of Bagnold's torque measurements $\left(M_{B a g}\right)$ divided by the torque for a boundary layer flow associated with a spinning disk, $M_{b l}$ given in equation (4.1) as a function of the Reynolds number. In the presentation of Bagnold's data, the fluid contribution has been included $\left(M_{B a g}=M^{\prime}+M_{\lambda}\right)$ for rotational speeds above 2.2 r.p.s. The inclusion of $M^{\prime}$ is most significant at the lowest concentrations, which correspond to the higher rotational speeds. The resulting torque ratio clearly increases with the concentration of solids.

For Bagnold's data using the glycerin mixture, the shear stress increased linearly with the shear rate. For the more viscous fluid, the torque would be expected to be dominated by the shear stress across the gap (equation (3.2)). In figure 7, the data for the glycerin mixture are also shown with the measurements scaled by $M_{\text {shear }}$ as given in equation (3.2).

The presence of particles in a Newtonian fluid is known to increase the effective viscosity. Since the original work by Einstein (1906), rheological studies of suspensions have developed relations for the ratio of the effective viscosity to the pure fluid Newtonian viscosity in terms of the solid fraction, maximum packing fraction, the shear rate and the polydispersity of the particles (Wildemuth \& Williams 1984; Liu \& Masliyah 1996; Krieger 1972. One such relation is the Krieger-Dougherty expression, which includes a dependency on the solid fraction $\phi$ and the maximum packing fraction $\phi_{m}$,

$$
\mu^{\prime}=\mu /\left(1-\phi / \phi_{m}\right)^{\eta \phi_{m}},
$$

The parameter $\eta$ is an empirical parameter that typically has a value of 2.5 , and $\phi_{m}$ is taken as 0.64 (Liu \& Masliyah 1996; Krieger 1972). Since the moment generated by the boundary-layer flow along the end plates, $M_{b l}$, scales with the square-root of the viscosity, the ratio of the torques for the water data (figure 7) could also be viewed as the square-root of the ratio of the effective viscosity to the pure fluid viscosity. Similarly, the moment generated by the shear flow in the gap, $M_{\text {shear }}$, scales with the 


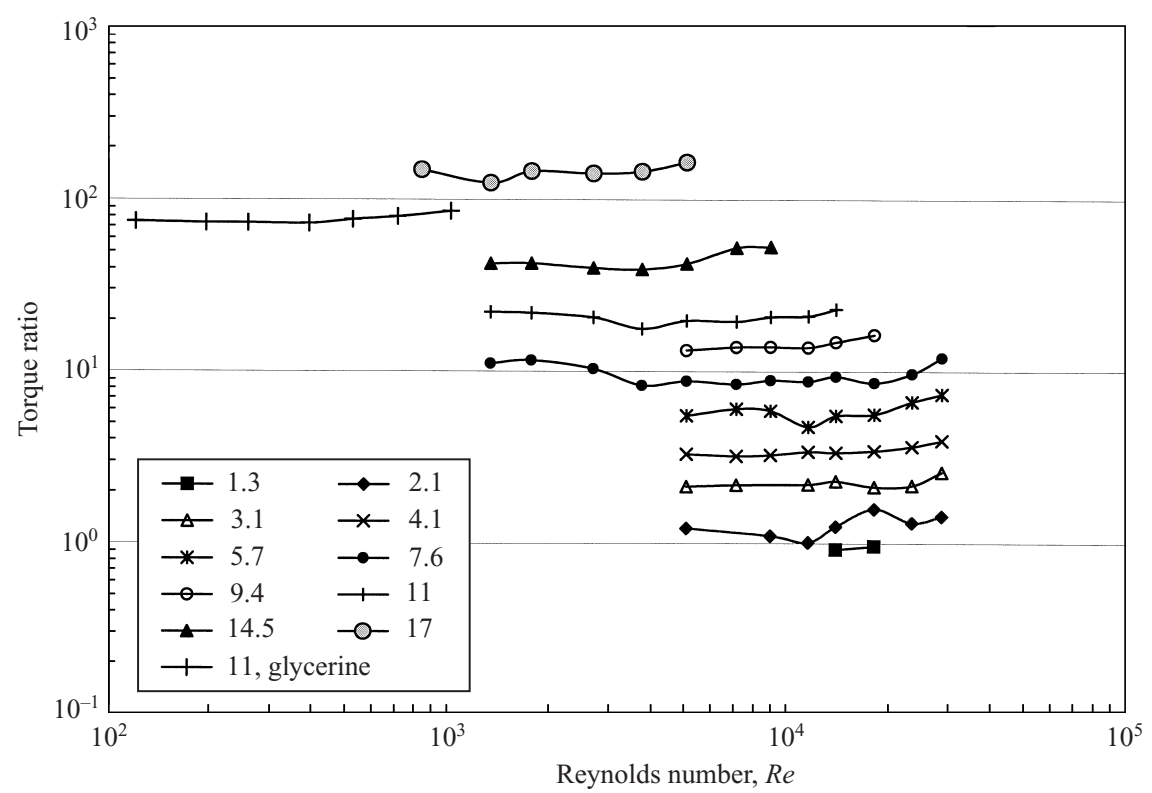

FIGURE 7. The ratio of Bagnold's measured torque using water, $M_{B a g}$, to torque calculated for laminar boundary layer flow over a spinning disk, $M_{b l}$ for different values of $\lambda$. The data for the glycerin mixture are for the ratio of $M_{B a g}$ to $M_{\text {shear }}$.

viscosity; hence, for the glycerin mixture, the torque ratio is equivalent to the ratio of the viscosities. Figure 8 presents the average of viscosity ratio as a function of the concentration for both the water and the glycerin data. Also shown are three lines for $\eta=2.5$ and $\phi_{m}=0.64, \eta=4.5$ and $\phi_{m}=0.64$, and $\eta=3.5$ and $\phi_{m}=0.68$. The curve $\eta=2.5$ and $\phi_{m}=0.64$ underpredicts the viscosity ratio; the curve for $\eta=4.5$ corresponds better with the water data, and the curve for $\eta=3.5$ and $\phi_{m}=0.68$ passes through the glycerin data point. The value of $\phi_{m}=0.68$ is used here because Bagnold's wax particles may have been more deformable than typically found for hard spheres. It should be noted that effective viscosity relations, such as equation (4.2) have been developed and compared with experimental data under low shear rate conditions. In addition, the effective viscosities shown in figure 8 could have been fitted with other functional relations that have appeared in the literature. The Krieger-Dougherty expression was used because it best represented the Bagnold data.

Figure 9 presents all of the experimental torque data by Bagnold, including the glycerin mixture, normalized by $\frac{1}{2} \rho \omega^{2} r_{o}^{3}\left(r_{o}^{2}-r_{i}^{2}\right)$. The resulting moment coefficient, $C_{m}=M_{B a g} /\left[\frac{1}{2} \rho \omega^{2} r_{o}^{3}\left(r_{o}^{2}-r_{i}^{2}\right)\right]$, is presented as a function of the effective Reynolds number based on gap, $R e^{\prime}=\rho r_{o} \omega t / \mu^{\prime}$, in which the viscosity is calculated as given in (4.2) using $\eta=3.5$ and $\phi_{m}=0.68$. This expression for the viscosity was chosen as the best fit for both the glycerin and the water data. The other values for the effective viscosity shift the data to the somewhat higher or lower Reynolds numbers. This simple representation for the effective viscosity assumes that the particles are uniformly distributed throughout the annulus (which is questionable because of the end effects) and that a single representation can be used over a wide range of shear rates and concentrations.

The three curves shown in figure 9 reflect different estimates of the torque assuming laminar single-phase Newtonian flow. The first is the moment coefficient for laminar 


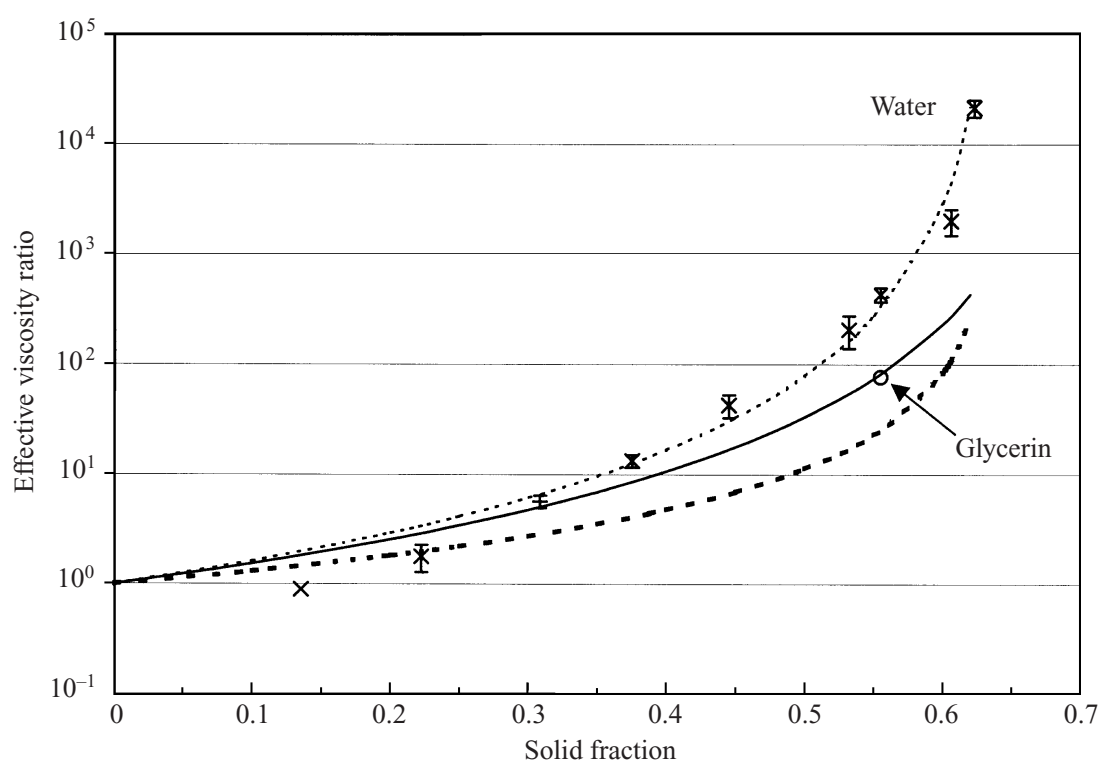

FIGURE 8 . The ratio of the effective viscosity to the pure fluid viscosity as a function of concentration. The experimental values from Bagnold (1954) are based on averaging the torque measurements shown in figure 7. The three lines are for effective viscosities using equation (4.2); the upper curve corresponds to $\eta=4.5$ and $\phi_{m}=0.64$; the middle curve to $\eta=3.5$ and $\phi_{m}=0.68$; and the lower curve to $\eta=2.5$ and $\phi_{m}=0.64$.

annular shear flow without end walls, which is calculated by normalizing the torque for Couette shear flow, equation (3.2), by $\frac{1}{2} \rho \omega^{2} r_{o}^{3}\left(r_{o}^{2}-r_{i}^{2}\right)$,

$$
C_{\text {shear }}=\frac{4 \pi \mu h \omega r_{o}^{2} r_{i}^{2}}{\frac{1}{2} \rho r_{o}^{3} \omega^{2}\left(r_{o}^{2}-r_{i}^{2}\right)^{2}}=\frac{8 \pi r_{i}^{2} h}{\left(r_{o}+r_{i}^{2}\right)\left(r_{o}-r_{i}\right)} R e^{-1} \text {. }
$$

The second dashed line in figure 9 is the moment coefficient from boundary layer theory for flow over a rotating disk coefficient, $C_{b l}$, computed from equation (4.1),

$$
C_{b l}=\frac{C^{\prime}(0) \pi \rho\left(\mu \omega^{3} / \rho\right)^{1 / 2}\left(r_{o}^{4}-r_{i}^{4}\right)}{\frac{1}{2} \rho r_{o}^{3} \omega^{2}\left(r_{o}^{2}-r_{i}^{2}\right)}=3.87 R e^{-1 / 2}\left(\frac{r_{o}-r_{i}}{r_{o}}\right)^{1 / 2}\left(\frac{r_{o}^{2}+r_{i}^{2}}{r_{o}^{2}}\right) .
$$

The solid line in the figure represents the sum of the two contributions to the moment, $C_{\text {shear }}+C_{b l}$. This summed moment coefficient suggests that the normalized torque depends inversely on the Reynolds number for low Reynolds number; for Reynolds numbers greater than approximately 100, the moment coefficient scales with $R e^{-1 / 2}$. Hence, there is a transition in the shear stress measurements from a linear dependence on the shear rate to a 1.5 power. The data for the highest concentration $(\phi=0.622)$ deviate from the other data; however, at the highest concentrations the particles may not move freely with the fluid and may arch across the gap. Bagnold alluded to problems with "contact melting" at high concentrations and high rotational speeds.

As the effective Reynolds number increases, the scaling of the thickness of the boundary layer along the end walls begins to approach the size of a particle. For these conditions, the particles may move from the high-shear boundary layer regime into the lower-shear central section of the annulus. As a result the effective viscosity ideas used in the presentation of figure 9 would no longer be appropriate. However, the highest values of $R e^{\prime}$ correspond with the lowest concentrations and the effective 


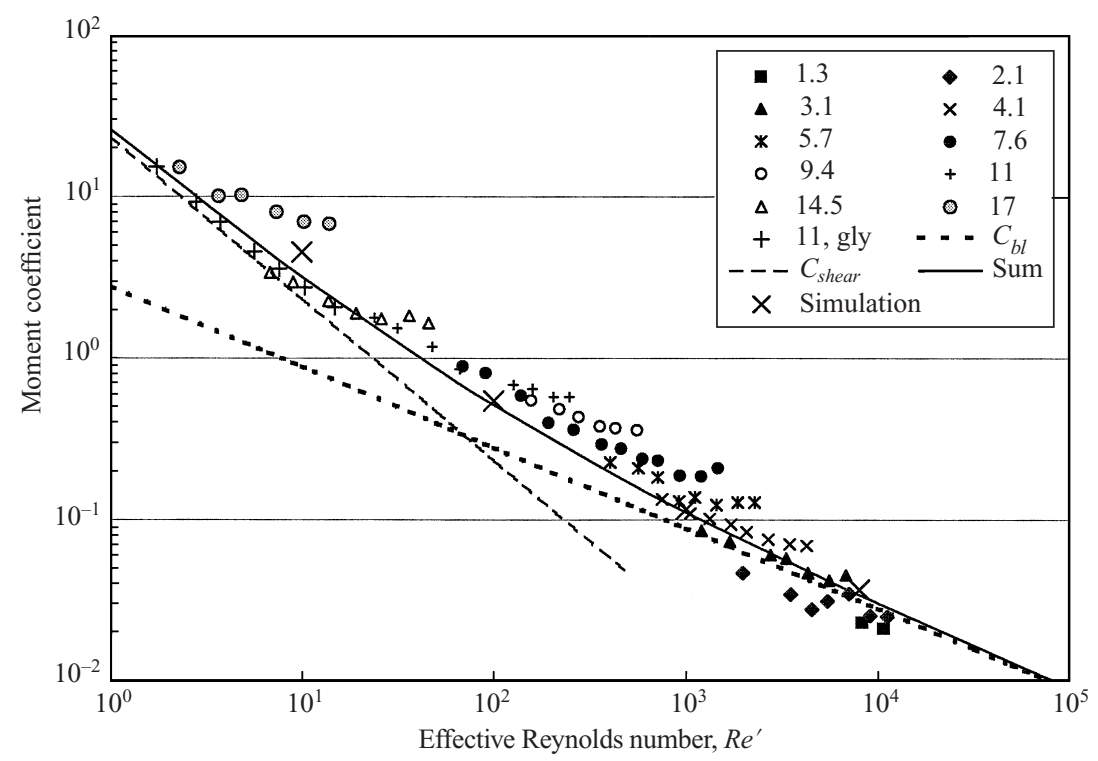

FIGURE 9. The moment coefficient, $C_{m}$ for all of Bagnold's data. The lines correspond to the moment coefficient for laminar shear flow in a gap, $C_{\text {shear }}$, for boundary layer flow over a rotating disk, $C_{b l}$, and for the sum, $C_{\text {shear }}+C_{b l}$. The $\times$ are results from the simulation.

viscosities closest to unity. If the effective viscosity were not used in presenting some of the data, such as for $\lambda \leqslant 3.1$, these points would shift from the left to the right of the theoretical line, but would not change the figure in a substantial way. Also note that as presented, the data at the highest effective Reynolds numbers indicate some flattening of the experimental moment coefficient suggesting an increased dependence on the shear rate. This deviation may also result from the assumption that the particles are uniformly distributed within the annulus. Hence, the method used in the presentation of figure 9 should be viewed as the simplest analysis that incorporates the essential features of the flow to estimate the measurements by Bagnold.

As a final check on the flow field generated by the movement of the top and bottom plates, a finite-element fluid dynamics program was used to solve the laminar NavierStokes equations for a Newtonian fluid subject to the no-slip boundary conditions along the bounding surfaces. A mesh of 34000 annular slices with elements in $5^{\circ}$ increments was used to replicate the Bagnold experimental device. The transverse section of the cell was divided into 28 by 36 elements of varying size; the nodes near the walls were placed at a maximum spacing of $0.1 \mathrm{~mm}$ to enable the calculation of the velocity gradients. The tangential velocity was fixed at $1 \mathrm{~m} \mathrm{~s}^{-1}$; the upper and lower surfaces moved with the outer wall. The Reynolds numbers were varied from 10 to 8000 by changing the viscosity. Calculations for flow Reynolds numbers above 8000 did not converge, suggesting a transition to turbulence. The value of $R e=8000$ coincides with the lower end of the data reported for pure water (see table 1). Beyond $R e=8000$, Bagnold reported that the shear stress increased with the square of the shear rate consistent with turbulent flow. As shown in figure 9, the range of effective Reynolds numbers was $1<R e^{\prime}<10000$. Although the finite element simulations were not used to calculate the turbulent pure-fluid flow field, the 

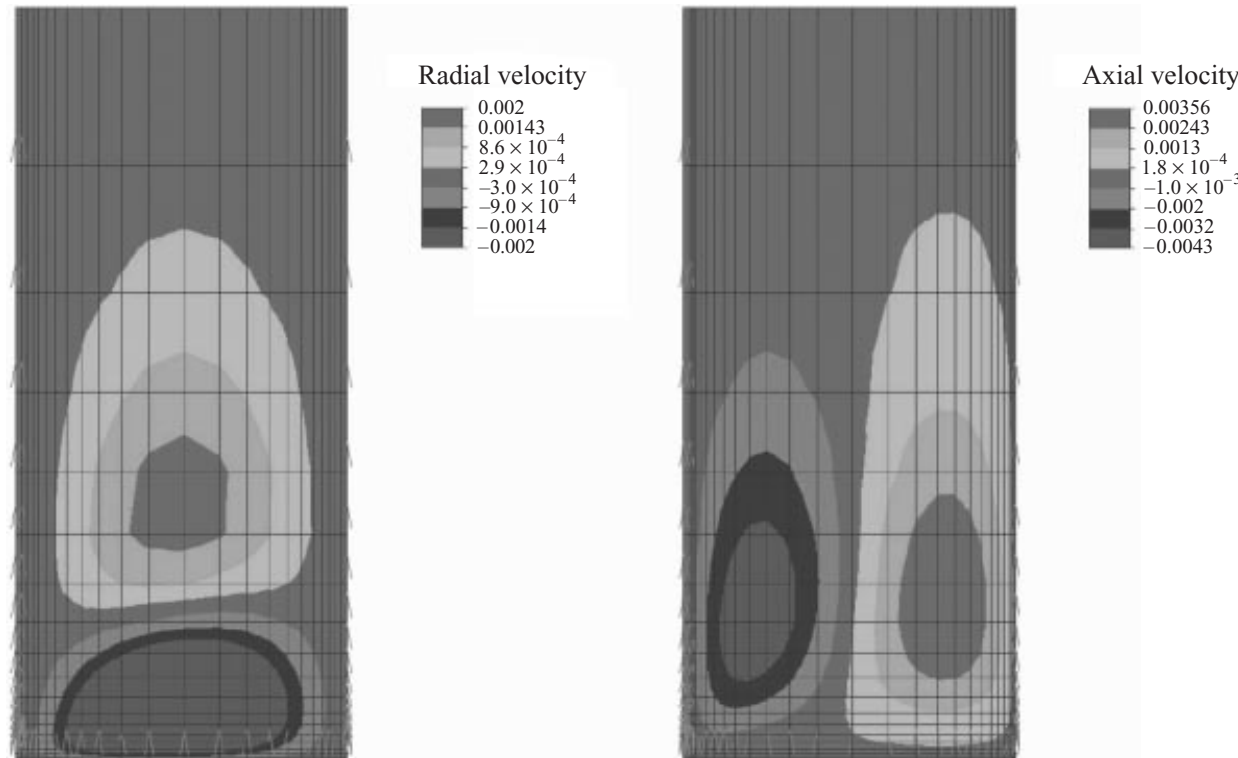

Figure 10. Calculations of the radial and axial velocity contours for a pure fluid at $R e=10$.

simulations covered nearly the entire range of the effective Reynolds numbers found in Bagnold's suspension experiments.

From the simulations, the moment coefficients were computed from measurements of the torque on the outer wall plus the top and bottom plates. These coefficients are shown as the large crosses on figure 9, indicating a good correspondence with the analytical predictions and the experimental values by Bagnold. Figures 10 and 11 show the velocity contours for the radial and axial velocity components at Reynolds numbers of 10 and 1000 , respectively. The left surface is the stationary inner wall and the right surface is the moving wall; the lower surface moves with the outer wall and the upper surface is the mid-height of the annulus. The velocities are scaled by the speed of the outer wall, $\omega r_{o}$. These contours clearly demonstrate the recirculation patterns and the outwardly directed flow along the lower end plate. For $R e=10$, the radial and axial velocities are less than $1 \%$ of the rotational speed of the outer cylinder. However, for $R e=1000$, the radial velocity field shows a boundary layer along with rotating end plate with a maximum speed of approximately $13 \%$ of the outer wall speed.

Figures 12 and 13 present the tangential velocity profiles for $R e=10$ and 1000 at several locations within the annulus. The figures also include the analytical result for flow in a gap without ends (equation (3.1)). For $R e=10$, the tangential profile at the mid-height of the channel $(z / h=0)$ is approximately equal to the analytical expression for flow without endplates. For $R e=1000$ the mid-height profile shows steeper gradients at the inner and outer walls. The average tangential velocity over the central region is approximately $46 \%$ of the rotational speed of the outer wall compared with $51 \%$ of the rotational speed for shear flow without ends; the average normalized tangential velocity decreases as the Reynolds number is increased. The tangential velocity profile is largely unchanged over more than $90 \%$ of the channel. At $z / h=0.488$ and 0.498 , the velocity profile is distorted because of the end conditions. 

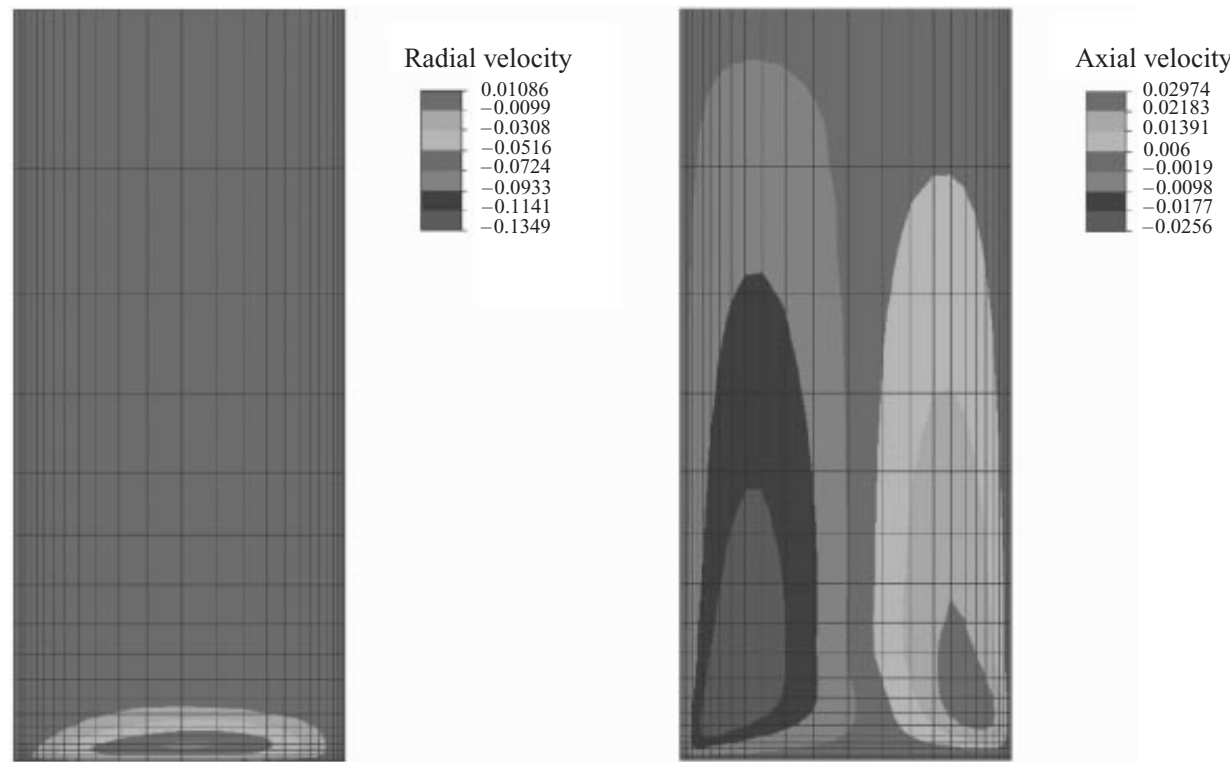

FIGURE 11. Calculations of the radial and axial velocity contours for a pure fluid at $R e=1000$.

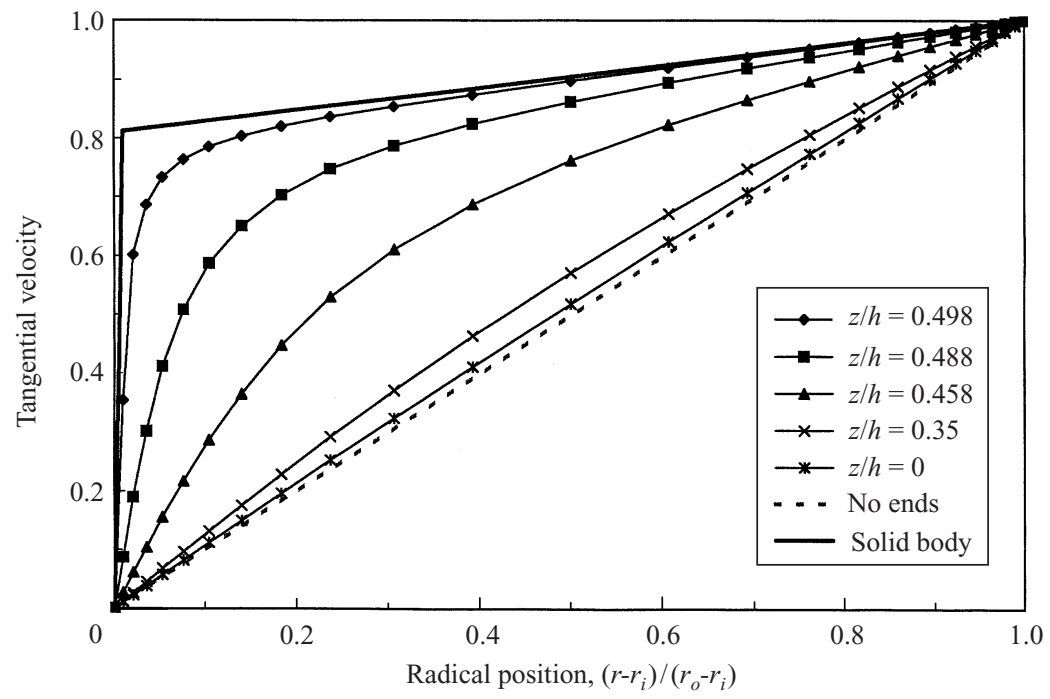

FIGURE 12 . The tangential velocity profiles for $R e=10$ at different axial locations.

At the lower Reynolds numbers, the variation of the velocity distribution occurs over a larger section of the annulus and is not confined to the thin boundary layer region.

Turning to the normal stresses measured by Bagnold, the experimental results appear to be more difficult to predict for several reasons. First, the normal stress values reported by Bagnold were the difference between the measured pressure with and without particles at a fixed rotational speed, as described in the presentation of equation (2.11). Although Bagnold reported the shear stress for a pure fluid, he did not report the corresponding values of the normal stresses, $P_{0}$. As noted by Bagnold, the pressure $P_{0}$ resulted from the centrifugal pumping motion in the end gaps and 


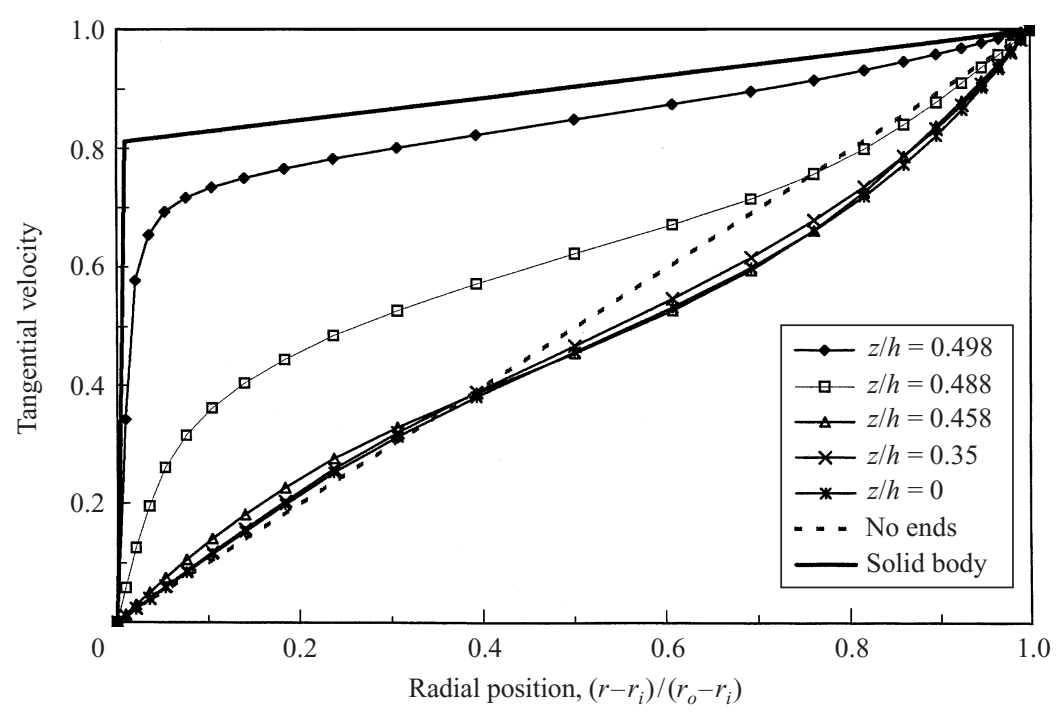

FIGURE 13 . The tangential velocity profiles for $R e=1000$ at different axial locations.

depended on the rotational speed. Second, the values of the normal stress for a pure fluid could not be simulated with the finite element code. Without particles, the flows were at Reynolds numbers within the turbulent flow regime. In addition, the calculations were done for a closed annulus and the absolute pressures within the experimental apparatus were dictated by the rotation of the drum and the flow within the end gaps. A final complication involves the technique to measure the normal stresses. The normal stress was averaged over the height of the annulus using a deformable membrane. Presumably this membrane was fixed to a frame at the top and bottom of the inner cylinder, creating a measurement that was more sensitive to the pressure in the center of the annulus than at the ends. As shown in the simulations, there are large gradients in the velocity field near the end plates especially at higher Reynolds numbers; as a result the pressure would also vary near the ends and would not be captured by the averaged measurement. It should also be noted that values of the normal stress differences were small and at most $2 \mathrm{~cm}$ of water.

Although the normal stress data could not be predicted, it is useful to examine the data in terms of a pressure coefficient, $C_{p}$, defined as the ratio of the net normal stress, $P_{\lambda}$, to the dynamic pressure $\frac{1}{2} \rho r_{o}^{2} \omega^{2}$,

$$
C_{p}=\frac{P_{\lambda}(\omega)}{\frac{1}{2} \rho \omega^{2} r_{o}^{2}}=\frac{P_{\text {gross }}(\omega, \lambda)-P_{0}(\omega, \text { tur } b)}{\frac{1}{2} \rho \omega^{2} r_{o}^{2}} .
$$

Figure 14 shows the pressure coefficient as a function of solid fraction for all of the data reported by Bagnold. The data are divided into several ranges of effective Reynolds number, $R e^{\prime}$. For $R e^{\prime}>200$, the pressure coefficient depends only on the solid fraction as indicated by the line drawn through the data points. At a fixed rotational speed the normal stress and the pressure coefficient increase with the solid fraction. For $100<R e^{\prime}<200$, the data show a weak dependence on the Reynolds number with the lower Reynolds numbers corresponding to a slightly larger value of the pressure coefficient. For $R e^{\prime}<100$, the pressure coefficient clearly depends on both the solid fraction and the Reynolds number. This low-Reynolds-number regime 


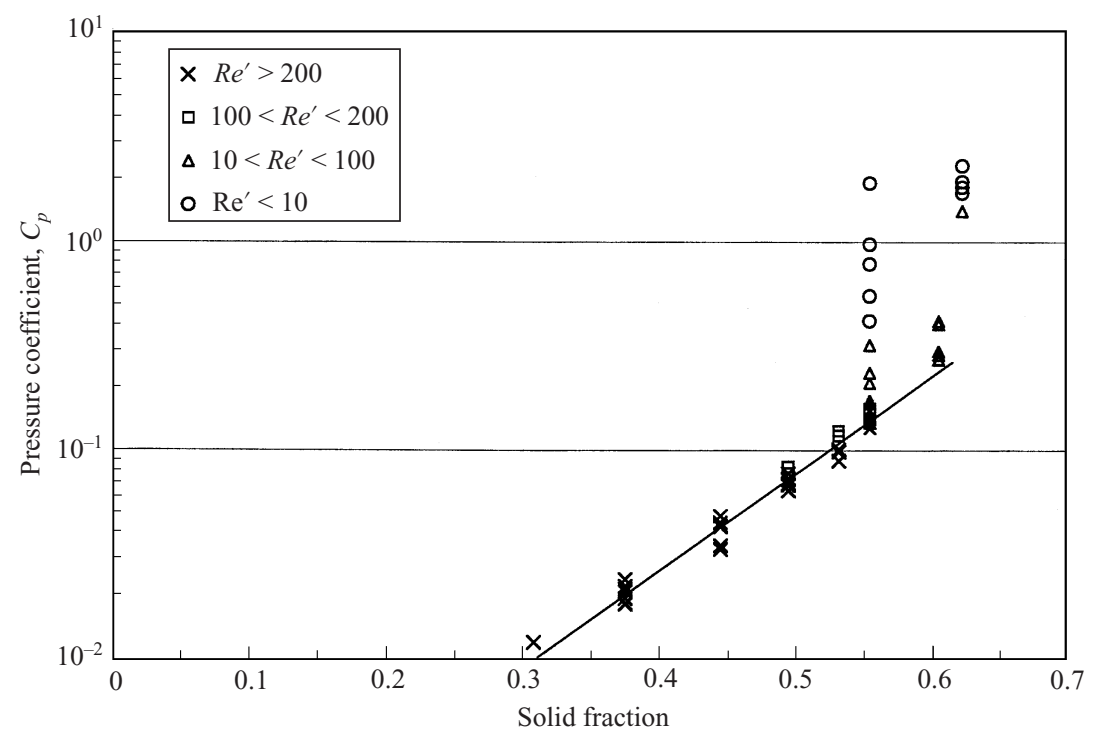

FIGURE 14. The pressure coefficient, $C_{p}$ as a function of solid fraction for ranges of $R e^{\prime}$.

covers the linear shear stress data for the glycerin mixture, as well as the normal stress measurements at $\phi=0.606$ and 0.623 .

\section{Closing remarks}

Although the ideas presented by Babnold (1954) have been essential to the development of modern theories in slurry transport, suspension rheology and granular material flows, the experiments were marred by the design of the experimental apparatus. Because the height of the annulus was only 4.6 times the size of the gap, the rotation of the end plates generated secondary vortices that contributed significantly to the torque measurements, especially at high rotational speeds. Bagnold's shear stress measurements and their dependence on the shear rate can be attributed to the effects of the end plates and not to particle interaction effects as Bagnold claimed. A critique of the normal stress measurements is harder due to uncertainty regarding the procedure used by Bagnold, but also appear to be due to changes in the flow patterns within the annulus.

Through physical arguments Bagnold stated that the shear and normal stresses should vary linearly with shear rate in the macro-viscous regime and vary quadratically due to increased particle-to-particle interactions in the grain-inertia regime. However, the experimental shear stress measurements do not show a quadratic dependence on shear rate; instead, the shear stress depends on the shear rate raised to the 1.5 power. The experimental torques of Bagnold are estimated using a boundary-layer analysis and finite-element simulations based on Newtonian flow with an effective viscosity that depends on solid concentration. For Reynolds numbers below 100, the torque results from linear shear flow across the annular gap. For larger Reynolds numbers the torque associated with the rotation of the end walls, which depends on the shear rate to the 1.5 power, is greater than for a linear shear flow. Hence, Bagnold's observed changes from the macro-viscous to the grain-inertia regime actually correspond to a variation from a linear shear flow to a flow dominated by the boundary layer along the rotating end walls. With a longer annular section, the contribution from the torque 
at the ends could have been reduced. Although Bagnold anticipated a problem with the end plates - "Errors due to non-uniform shear strain at the ends of the annular space were unavoidable and difficult to assess" Bagnold (1954) - the earlier work by Couette (1890), Mallock (1896), Wendt (1933) and Taylor (1936a) could have been used to estimate the extent of the problem.

A question lingers as to why Bagnold's work has not received a detailed review before now. One reason is that by 1954 Bagnold was a Fellow of the Royal Society; hence, his work could be published in the Proceedings without review. The experimental apparatus, however, was similar to that used in the earlier laminar-to-turbulent transition studies as described in $\S 3$. Taylor had been critical of the work by both Mallock and Wendt (Taylor 1923, 1936b) because of the relatively short annuli used in their experiments. Bagnold's experimental section $(h / t=4.6)$ was shorter than that used by either Mallock $(h / t=8.8)$ or Wendt $(h / t=28)$.

Hence, it is surprising that the same criticism was not extended to Bagnold because Bagnold had scientific interactions with Taylor, at least in the early part of Bagnold's career. The papers by Bagnold in the 1930s in the Proceedings were all communicated through Taylor (Bagnold 1936b, 1937, 1938). Bagnold's 1936 paper appeared in the same volume as the two papers by Taylor on flow between rotating cylinders (Taylor $1936 a, b)$; in fact, there was but a single paper physically separating them. In the "Introduction" to the 1941 publication of The Physics of Blown Sand and Desert Dunes, Bagnold thanked Professor G. I. Taylor for his "encouragement and help on many occasions". In 1946, Bagnold published a paper on the interaction of a shallow wave and a sandy bed. The paper was published, presumably without review, when Bagnold was a Fellow of the Royal Society (Bagnold 1946). In the Proceedings, the paper was immediately followed by a note, written by Taylor, presenting a theoretical derivation of the conditions under which a grain might be expected to roll as a result of shear forces (Taylor 1946). Taylor compared his prediction with Bagnold's empirical relation and experimental data. Even in the 1954 paper, Bagnold included a passing reference to Taylor regarding a discussion of the forces generated as a particle collides with a surface. Despite these interactions, Bagnold was apparently unaware of Taylor's work on flow transitions in annular shear flows, and perhaps Taylor was also unaware of the details of Bagnold's experimental apparatus. After the publication of Bagnold (1954), there are no further references to Taylor in Bagnold's technical papers, or to Bagnold in Taylor's papers.

In the "Concluding Remarks" contained in the Collection of Hallmark Papers by R. A. Bagnold (Bagnold 1988), Bagnold offers a two-page essay on the "Truth" and its role in the development of scientific or engineering concepts. He concludes by writing that with his friend Luna B. Leopold (head of the Water Resources Division of the United States Geological Survey), "we both saw the need to 'stir the pool of complaisant tradition with the stick of reality" concerning traditional approaches to sediment transport. The purpose of this article has been to stimulate discussions and questions of the widely cited studies by Bagnold. It appears that although the 1954 experiments were visionary, the experimental facility and procedure were flawed. New experiments on the rheological properties of liquid-solid flows that avoid the problematic features found in Bagnold's design are needed.

The authors obtained from Churchill College Archives Centre, Cambridge, UK, the Catalogue of the Papers and Correspondence of Brigadier Ralph Alger Bagnold, F.R.S. compiled in 1992 by Timothy E. Powell and Peter Harper of the University of Bath. The 61-page catalogue lists all of the materials held by Churchill College 
including hundreds of maps of North Africa, military expeditions, travel documents and photographs. However, the catalogue notes on page 6, "Bagnold's scientific research is less well documented; most of the material dates from the post-war period and the work best represented is Bagnold's later research on random distributions". In the catalogue, we did not find anything related to Bagnold's rheological studies. We also obtained the Catalogue of the Papers and Correspondence of Sir Geoffrey Ingram Taylor, O.M.F.R.S. (1886-1975) by Jeannine Alton, Harriot Weiskittel, Julia LathamJackson from the National Cataloguing Unit for the Archives of Contemporary Scientists, University of Bath, UK. Although the 102-page catalogue contains an extensive list of scientific correspondence with Taylor, there is only one reference to R. A. Bagnold. In 1970, Bagnold sent a letter to Taylor; the catalogue has a notation "water 'boils" " next to the listing, which presumably is unrelated to the suspension work.

The authors thank Professors Ronald F. Scott, Rolf H. Sabersky and Norman H. Brooks for their interest and assistance in writing this paper, and to G. G. Joseph for the use of his library. M.L.H. also thanks Professor D. A. Kaminski for suggesting a review article on liquid-solid flows. We acknowledge the support of NASA through grant NAF3-2358, the National Science Foundation through grant CTS-9908430, and UNAM-PAPIIT grant IN-103900.

\section{REFERENCES}

Bagnold, R. A. 1936 a Libyan Sands: Travel in a Dead World. London: Hodder and Stoughton. Bagnold, R. A. $1936 b$ The movement of desert sand. Proc. R. Soc. Lond. A 157, 594-620.

Bagnold, R. A. 1937 The size-grading of sand by wind. Proc. R. Soc. Lond. A 163, 250-264.

Bagnold, R. A. 1938 The movement of sand storms. Proc. R. Soc. Lond. A 167, 282-291.

Bagnold, R. A. 1941 The Physics of Blown Sand and Desert Dunes. New York: William Morrow \& Co.; republished by Methuen \& Co., London, 1954, 1960, 1971; to be republished by the Blackburn Press, 2001.

BAGNOLD, R. A. 1946 The motion of waves in shallow water. Interaction between waves and sand bottoms. Proc. R. Soc. Lond. A 187, 1-15.

BAGNOLD, R. A. 1954 Experiments on a gravity-free dispersion of large solid spheres in a Newtonian fluid under shear. Proc. R. Soc. Lond. A 225, 49-63.

BAGnold, R. A. 1956 The flow of cohesionless grains in fluids. Proc. R. Soc. Lond. A 249, 235-297.

BAGNOLD, R. A. $1966 a$ An approach to the sediment transport problem from general physics. Tech. Rep. 422-I. US Geological Survey professional paper.

BAgnold, R. A. $1966 b$ The shearing and dilation of dry sand and the 'singing' mechanism. Proc. R. Soc. Lond. A 295, 219-232.

Bagnold, R. A. 1973 The nature of saltation and of 'bed-load' transport in water. Proc. R. Soc. Lond. A 332, 473-504.

BAGNold, R. A. 1980 An empirical correlation of bedload transport rates in flumes and natural rivers. Proc. R. Soc. Lond. A 372, 453-473.

BAgnold, R. A. 1988 The Physics of Sediment Transport by Wind and Water: A Collection of Hallmark Papers by R. A. Bagnold (ed. C. R. Thorne, R. C. MacArthur \& J. B. Bradley). New York: The American Society of Civil Engineers.

Bagnold, R. A. 1990 Sand, Wind and War: Memoirs of a Desert Explorer. University of Arizona Press.

Coles, D. 1964 Transition in circular Couette flow. J. Fluid Mech. 21, 385-425.

Couette, M. 1890 Etudes sur le frottement des liquides. Ann. Chim. Phys. 6, 433-510.

EINSTEIN, A. 1906 Calculation of the viscosity-coefficient of a liquid in which a large number of small spheres are suspended in irregular distribution. Ann. Physik 19, 286-306, English translation in Investigation on the Theory of Brownian Movement, Dover, 1956. 
Gelhar, L. W., Schriek, W. \& Benham, F. A. 1996 Secondary motion and turbulence in circular Couette flow.Rep. R66-21. Ralph M. Parsons Laboratory for Water Resources and Hydrodynamics, MIT, Cambridge, MA.

Hanes, D. M. \& InMAn, D. L. 1985 Observations of rapidly flowing granular-fluid materials. J. Fluid Mech. 150, 357-380.

Hill, H. M. 1966 Bed forms due to a fluid stream. J. Hydraul. Div., Proc. ASCE 92, 127-143.

Hutter, K. \& Rajagopal, K. R. 1994 On flows of granular materials. Continuum Mech. Thermodyn. 6, 81-139.

Kenn, M. J. 1990 Ralph Alger Bagnold. Biogr. Mem. Fell. R. Soc. Lond. 37, 55-68.

Koschmieder, E. L. 1993 Bénard Cells and Taylor Vortices. Cambridge University Press.

Krieger, I. M. 1972 Rheology of monodisperse latices. Adv. Colloid Interface Sci. 3, 111-136.

LiU, S. \& Masliyah, J. H. 1996 Rheology of Suspensions (ed. L. L. Schramm), chap. 3, pp. 107-176. Washington, DC: American Chemical Society.

Mallock, A. 1896 Experiments on fluid viscosity. Phil. Trans. R. Soc. Lond. A 183, 41-56.

Potapov, A. V., Hunt, M. L. \& CAmpbell, C. S. 2001 Liquid-solid flows using smoothed particle hydrodynamics and the discrete element method. Powder Technol. 116, 204-213.

Prasad, D. \& KytömaA, H. K. 1995 Particle stress and viscous compaction during shear of dense suspensions. Intl J. Multiphase Flow 21, 775-785.

ReYNOLDS, O. 1885 On the dilatancy of media composed of rigid particles in contact. Phil. Mag. 20, 469-481.

SAGAN, C. \& Bagnold, R. A. 1975 Fluid transport on earth and Aeolian transport on Mars. Icarus 26, 209-218.

Savage, S. B. \& JefFrey, D. J. 1981 The stress tensor in a granular flow at high shear rates. J. Fluid Mech. 110, 255-272.

Savage, S. B. \& McKeown, S. 1983 Shear stresses developed during rapid shear of concentrated suspensions of large spherical particles between concentric cylinders. J. Fluid Mech. 127, 453-472.

Schlichting, H. 1955 Boundary Layer Theory. McGraw Hill.

Schultz-Grunow, F. 1959 Zur Stabilitat der Couette-Stromung. Z. Angew. Math. Mech. 39, 101110.

ScotT, R. F. 1963 Principles of Soil Mechanics. Addison-Wesley.

Shaw, W. B. K. 1945 Long Range Desert Group: The Story of its Work in Libya. 1940-1943. Collins.

Shibata, M. \& MeI, C. C. 1986 Slow parallel flows of a water-granule mixture under gravity. Part I: continuum modeling. Acta Mechanica 63, 179-193.

TAYLOR, G. I. 1923 Stability of a viscous liquid contained between two rotating cylinders. Phil. Trans. R. Soc. Lond. A 223, 289-343.

TAYLOR, G. I. $1936 a$ Fluid friction between rotating cylinders I. - torque measurements. Proc. R. Soc. Lond. A 157, 546-564.

TAYLOR, G. I. 1936b Fluid friction between rotating cylinders II. - distribution of velocity between concentric cylinders when outer one is rotating and inner one is at rest. Proc. R. Soc. Lond. A 157, 565-578.

TAYLOR, G. I. 1946 Note on R. A. Bagnold's empirical formula for the critical water motion corresponding with the first disturbance of grains on a flat surface. Proc. R. Soc. Lond. A 187, $16-18$.

Terzaghi, K. 1943 Theoretical Soil Mechanics. Wiley.

Thorne, C. R. \& Soar, P. 1996 R. A. Bagnold: A biography and extended bibliography. Earth Surface Process. Landforms 21, 987-991.

WENDT, F. 1933 Turbulente Stömungen zwischen zwei rotierenden konaxialen Zylindern. Ing.-Arch. 4, 577-595.

Wildemuth, C. R. \& Williams, M. C. 1984 Viscosity of suspensions modeled with a shear-dependent maximum packing fraction. Rheological Acta 23, 675-635. 